\title{
Power-Budget Equations and Calibration Factors for Fish Abundance Estimation Using Scientific Echo Sounder and Sonar Systems
}

\author{
Per Lunde ${ }^{1,3, *}$ and Rolf J. Korneliussen ${ }^{2,3}$ \\ 1 Department of Physics and Technology, University of Bergen, P.O. Box 7803, N-5020 Bergen, Norway \\ 2 Institute of Marine Research, P.O. Box 1870, Nordnes, N-5817 Bergen, Norway; rolf.korneliussen@imr.no \\ 3 Christian Michelsen Research AS, P.O. Box 6031, Postterminalen, N-5892 Bergen, Norway \\ * Correspondence: per.lunde@uib.no; Tel.: +47-55582786
}

Academic Editor: Jens Martin Hovem

Received: 29 February 2016; Accepted: 4 July 2016; Published: 9 July 2016

\begin{abstract}
Acoustic methods used in fish abundance estimation constitute a key part of the analytic assessment that makes the basis for abundance estimation of marine resources. The methods rely on power-budget equations and calibrated systems. Different formulations of power-budget equations and calibration factors have been proposed for use in scientific echo sounder and sonar systems. There are unresolved questions and apparent inconsistencies in prior literature related to this field. A generic (instrument independent) and unifying theory is presented that attempts to explain the different power-budget and calibration factor formulations proposed and used in prior literature, and how these are mutually related. Deviations and apparent inconsistencies in this literature appear to be explained and corrected. This also includes different (instrument specific) formulations employed in important modern scientific echo sounder systems, and their relationship to the generic theory of abundance estimation. Prior literature is extended to provide more complete power-budget equations for fish abundance estimation and species identification, by accounting for echo integration, electrical termination, and the full range of electrical and acoustical echo sounder parameters. The expressions provide a consistent theoretical basis for improved understanding of conventional methods and instruments used today, also enabling improved sensitivity and error analyses, and correction possibilities.
\end{abstract}

Keywords: fisheries acoustics; single-target backscattering; volume backscattering; echo integration

\section{Introduction}

Acoustic methods are widely used for estimating fish abundance [1-28], and constitute a key part of the analytic assessment that makes the basis for international regulations of marine resources. For fish aggregated in schools or layers, echo integration $[7,8]$ supported by biological sampling, is the most common method used in oceanic surveys [3]. Fish abundance is measured using narrowband signals typically in the $10-120 \mathrm{kHz}$ range, whereas species identification of fish and quantification of zooplankton are based on multi-frequency signals extended to $500 \mathrm{kHz}$ or higher. The acoustic methods rely on calibrated systems $[9,10]$ and power-budget equations.

In oceanic surveys, a power-budget equation for multi-target (volume) backscattering $[6,11-16]$ is typically used to measure volume backscattering from aggregations of fish or zooplankton. In terms of this equation, the volume backscattering coefficient $s_{v}$ is measured for a sequence of thin spherical shell "ping volumes", $V_{p}$, at increasing range. The sequence of $s_{v}$ measurements is integrated over the range of an observation volume $V_{o b s}[6,11,16]$, to give the fish density in $V_{o b s}, \rho_{a}$ [17], in terms of an echo-integrator equation $[3,6,8,10,16]$. 
Prior to survey operation, a related power-budget equation for single-target backscattering $[6,11-16]$ is used for at-sea calibration of the echo sounder using a standard target $[3,6,9,10]$. The same power-budget equation for single-target backscattering is used to measure the backscattering cross section, $\sigma_{b s}$, or equivalently, the target strength, $T S$, of individual fish.

Different formulations of power-budget equations and calibration factors have been presented for use in scientific echo sounder and sonar systems.

Clay and Medwin [1], and later Medwin and Clay [5], derived expressions corresponding to $s_{v}$ that account for sound propagation in the sea, in terms of echo integration of the free-field acoustic pressure signals at the centre of the transmitting and receiving transducer's front surface [16].

Dalen and Nakken [8] proposed an empirical echo-integrator equation for estimation of $\rho_{a}$, involving a calibration factor to be determined by at-sea calibration using a standard target (e.g., a metal sphere). The expression was modified by Foote et al. [10], cf. also [3,4,6].

Simrad [11] extended Clay and Medwin's [1,5] analyses by accounting for the transmitting and receiving transducer responses. Power-budget equations for $\sigma_{b s}$ and $s_{v}$ were given on average power form, in addition to expressions postulated for two calibration factors used in the Simrad EK500 echo sounder system [11]. The formulation applies to certain conditions of electrical termination of the transducer [16] (cf. Section 4.1). The Simrad formulation constitutes an important basis for the methods used in fish abundance measurement today. Simrad's derivation of the expressions for $\sigma_{b s}$ and $s_{v}$ was summarized by Korneliussen [12].

Demer and Renfree [14] also used expressions for $\sigma_{b s}$ and $s_{v}$ that correspond to those given in [11]. Related power-budget equations were postulated by Simmonds and MacLennan [6] (see their Equations (3.13) and (3.15)). However, when these are re-arranged to yield expressions for $\left\langle\sigma_{b s}\right\rangle$ (the expected value of $\left.\sigma_{b s}\right)$ and $s_{v}$, the resulting expressions may be shown to differ somewhat from those given in [11], and power flow balance is not quite preserved.

In a more detailed derivation, Pedersen [13] extended Simrad's [11] analysis by-in the power-budget equations for $\sigma_{b s}$ and $s_{v}$-including a factor accounting for arbitrary electrical termination of the transducer.

Lunde et al. [16] further developed the power-budget equations that are given on average power form by [11,13], to account for echo integration. $\sigma_{b s}, s_{v}$, and $\rho_{a}$ were expressed in terms of time integration of the transmitted and received voltage signals (echo integrals). The resulting expressions were shown to represent a consistent generalization of the echo integration expressions derived by Clay and Medwin [1,5] for "in-water" pressure signals.

For the more recent generation of Simrad scientific and fisheries echo sounder and sonar systems, such as EK60, ES60, ME70 and MS70, an apparently different set of power-budget equations is used [15]. Ona et al. [15] postulated equations for $\sigma_{b s}$ and $s_{v}$ that are used in these systems [18-21]. A derivation of similar power-budget equations and calibration factors as used in $[11,15,18-21]$ was given by Lunde and Korneliussen [22]. An alternative derivation of the $\sigma_{b s}$ and $s_{v}$ expressions being postulated in [15] was presented in [23].

There are unresolved questions and some apparent inconsistencies related to the prior literature in this field.

Firstly, in [11], expressions have been postulated for two calibration factors, "TS transducer gain" and " $S_{v}$ transducer gain", being determined by echo sounder calibration. However, neither definition, nor the mathematical relationship between these calibration factors and the power-budget equations for $\sigma_{b s}$ and $s_{v}$ given in [11], are available from the literature. That is, no power-budget equations for $\sigma_{b s}$ and $s_{v}$, expressed in terms of the two calibration factors, are given. It can be shown [22] that the expressions for the two calibration factors postulated in [11] cannot be readily derived from the average power formulation of the power-budget equations given in [11].

Secondly, ref. [11] states that "the TS-measurement is based on the peak value of the echo samples in the sphere echo, whereas the $s_{A}$-measurement is based on integration (averaging) of the echo samples". This difference between $\sigma_{b s}$ and $s_{v}$ measurements is not reflected in the power-budget 
equations given in [11]. This situation strongly indicates that the power-budget equations for $\sigma_{b s}$ and $s_{v}$ that are actually used in EK500, and which are presumed to be expressed in terms of the two EK500 calibration factors, may not be exactly the same as those given in [11].

Thirdly, the expression for $s_{v}$ postulated by [15] differs from those given by other authors $[6,11,13,14,16]$, and is not consistent with the expression given e.g., in [11]. In particular this concerns a parameter $S_{a, \text { corr }} \equiv 10 \log \left(s_{a, \text { corr }}\right)$ introduced by [15] in their logarithmic expression for $s_{v}$ [22]. The ratio of $s_{v}$ as given by [11] and [15] is not equal to 1, but $s_{a, c o r r}^{-2}$. It follows [22] that the power-budget equations as given in [15] cannot be derived directly from the power-budget equations given in [11].

Fourthly, the set of power-budget equations given in [15] involves two calibration factors, "Axial transducer gain" and "Integration correction", being determined by echo sounder calibration. In [18-21] these are referred to as "Gain" and " $S_{a}$ correction". In the literature available for these instruments [15,18-21], the calibration factors are not defined, nor given by any expressions.

Fifthly, the derivation given in [23] of the power-budget equations for $\sigma_{b s}$ and $s_{v}$ being postulated in [15], appears to be incomplete on essential points, such as with respect to the $s_{a, \text { corr }}$ parameter. In [23], $s_{a, \text { corr }}$ is introduced ad hoc by replacing the nominal pulse duration, $\tau_{p}$ (in terms of the terminology used here), with an effective pulse duration, defined as $\tau_{e f f} \equiv \tau_{p} s_{a, c o r r}^{2}$. The mathematics and arguments used are not clear, and the expression for $s_{v}$ given by [23] deviates from those given by other authors $[6,11,13,14,16]$.

The unclear situation connected to (i) the deviating and apparently inconsistent expressions given in the literature in this field; and (ii) the sparse documentation on the power-budget equations and calibration factors actually employed in widely used echo sounder and sonar systems, has caused uncertainty and confusion among users. The equations used for international regulations of marine resources, including their theoretical basis, should preferably be known and documented in available literature. Moreover, the equations should be sufficiently complete to enable accurate and reliable analyses of error and system drift.

The objective of the present paper is to formulate a generic (instrument independent) and unifying theory of the power-budget and echo-integrator equations for $\sigma_{b s}, s_{v}$, and $\rho_{a}$, that is capable of explaining the different power-budget and calibration factor formulations used in the literature, and how these different formulations are mutually related. By "unifying" is meant "giving the connection and relationships between different (and apparently diverging) formulations".

Table 1 gives an overview of the five formulations addressed here. Based on the average power formulation of the $\sigma_{b s}$ and $s_{v}$ equations given in $[13,16]$ (denoted "Formulation $\mathrm{A}^{\text {") }}$, an echo integrator formulation is derived for narrowband signals (denoted "Formulation B"). Expressions are presented for a calibration factor that can be measured using current methods for at-sea echo sounder calibration employing a metal sphere. As an alternative, $\sigma_{b s}$ and $s_{v}$ are also expressed directly in terms of the basic quantities being measured in calibration. The corresponding expression for $\rho_{a}$ is derived, applicable to both alternatives.

As an application of this generic theory, alternative power-budget and calibration factor formulations are derived for echo sounders based on using "peak voltage echo integration" in single-target TS measurements, instead of the full echo waveform itself [11,18-21]. Three equivalent formulations are derived, denoted "Formulation $C$ ", " $\mathrm{D}$ ", and “ $\mathrm{E}$ ", respectively. These are all equivalent to Formulation $\mathrm{B}$, and fully valid approaches, for instruments using this signal processing strategy.

The resulting expressions are shown to be closely related to the equations given for two widely used and important families of scientific echo sounder and sonar systems; (i) the Simrad EK500 [11] and (ii) the more recent Simrad EK60, ES60, ME70 and MS70 [15,18-21] systems. By using the equivalent Formulations $\mathrm{C}, \mathrm{D}$, and $\mathrm{E}$, the deviations and apparent inconsistencies between the expressions given in refs. $[15,23]$ and those of refs. $[11,13,16]$ seem to be explained and resolved. This includes the $S_{a, c o r r}$ parameter, as well as definitions of, relationships between, and explanation for use of the various 
calibration factors involved in the two echo sounder systems, and how these are mathematically related to the power-budget equations of the respective instruments.

Table 1. Alternative and equivalent formulations of power budget equations for measurement of $\sigma_{b s}$ and $s_{v}$. Calibration factors referred to in the table are defined in Sections 2 and 3.

\begin{tabular}{|c|c|c|c|c|c|}
\hline $\begin{array}{c}\text { Form } \\
\text { No. }\end{array}$ & $\begin{array}{l}\text { Type of } \\
\text { Formulation }\end{array}$ & $\begin{array}{l}\text { Generic or } \\
\text { Not }\end{array}$ & $\begin{array}{l}\text { Calibr. } \\
\text { Factors }\end{array}$ & Key Characteristics & Refs. \\
\hline A & Average power & $\begin{array}{l}\text { Instrument } \\
\text { independent } \\
\text { (generic) }\end{array}$ & None & $\begin{array}{l}\text { - Not directly suitable for signal } \\
\text { processing (echo integration) in this } \\
\text { formulation }\end{array}$ & $\begin{array}{l}{[11]} \\
{[13]} \\
{[16]}\end{array}$ \\
\hline B & Echo integration & $\square^{\prime \prime}-$ & $G_{0}$ & $\begin{array}{l}\text { - Full waveform echo integration in } \\
\sigma_{b s} \text { and } s_{v} \text { measurements }\end{array}$ & [22] \\
\hline $\mathrm{C}$ & $\square^{\prime \prime}-$ & $\begin{array}{l}\text { Instrument } \\
\text { dependent }\end{array}$ & $\begin{array}{c}G_{0, e f f} \\
G_{0}\end{array}$ & $\begin{array}{l}\text { - "Peak voltage echo integration" in } \\
\sigma_{b s} \text { measurement } \\
\text { - Full waveform echo integration in } s_{v} \\
\text { measurement } \\
\text { - Equations related to Simrad EK500 } \\
\text { type of echo sounder system [11] }\end{array}$ & [22] \\
\hline $\mathrm{D}$ & 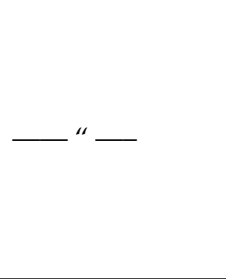 & $\square^{\prime \prime}-$ & $\begin{array}{l}G_{0, e f f} \\
s_{a, \text { corr }}\end{array}$ & $\begin{array}{l}\text { - "Peak voltage echo integration" in } \\
\sigma_{b s} \text { measurement } \\
\text { - Full waveform echo integration in } s_{v} \\
\text { measurement } \\
\text { - Equations related to Simrad EK60, } \\
\text { ES60, ME70, and MS70 type of } \\
\text { scientific echo sounder and sonar } \\
\text { systems [15,18-21] }\end{array}$ & [22] \\
\hline $\mathrm{E}$ & —“ & $\square^{\prime \prime}-$ & $\begin{array}{c}G_{0, e f f} \\
\tau_{e f f}\end{array}$ & $\begin{array}{l}\text { - "Peak voltage echo integration" in } \\
\sigma_{b s} \text { measurement } \\
\text { - Full waveform echo integration in } s_{v} \\
\text { measurement }\end{array}$ & [22] \\
\hline
\end{tabular}

Formulation B is derived and employed here as the "generic and unifying theory" since it can be used to derive the connections and relationships between Formulation A and Formulations C, D, and $\mathrm{E}$, including explanation of the different calibration factors. It represents a necessary key approach to derive, explain, and understand Formulations C-E. That is, to reveal and explain the formulations and expressions underlying the Simrad EK500, EK60, etc., scientific echo sounder and sonar systems. In Formulation B, full waveform echo integration (here denoted [tivs], cf. Section 2.3.1) is used for both $\sigma_{b s}$ and $s_{v}$ measurements (i.e., in calibration and field measurements), since that represents a key approach to reveal and explain the diverse echo integration methods and calibration factors that are in use in modern scientific echo sounder and sonar systems. The objective is thus not to find a "best" or preferred method or formulation of power budget equations and calibration factors for fish abundance estimation. The intention and objective is to address the challenges encountered and inconsistencies identified in the literature on modern scientific echo sounder and sonar systems, and present a theory that explains and resolves these inconsistencies.

The present paper represents a condensed, partly reformulated, and extended version of a technical report [22], in which further details on the mathematical derivations can be found. Extensions of [22] relate to placement of the theory and results e.g., in the context of recent literature, and a more comprehensive derivation of the echo integration formulation (Section 2.3.1).

The main contribution of the present paper is the generic and unifying theory for echo sounders operated in their linear (small-amplitude) range (Formulation B), used to reveal the relationship between, and to correct, some diverging and inconsistent expressions given in prior literature [11,15,18-21,23]. 


\section{Theory}

\subsection{Assumptions}

The analysis is based on the following assumptions for the echo sounder and its environment (cf. [16] and references therein):

(a) the monostatically operated transducer is passive, reversible, and reciprocal;

(b) the transmit voltage amplitude is sufficiently small to avoid nonlinear effects in the electroacoustic transducer and electronics (i.e., the transducer and electronics are operated in their linear ranges);

(c) the fluid medium (seawater) is homogeneous, with constant density and sound velocity;

(d) the amplitudes of the transmitted sound pressure signals are sufficiently small so that finite-amplitude sound propagation effects in seawater can be neglected;

(e) targets are in the far field of the transducer;

(f) possible nonlinear effects in the scattering process at the target itself (involving e.g., fish with gas-filled swim-bladder), can be neglected, so that the linear backscattering theory applies;

(g) the volume backscattering coefficient can be calculated as a sum of backscattering cross sections (i.e., intensities) per unit volume;

(h) the scattering objects are uniformly distributed in the observation volume, with

(i) random phases of the scattered echoes (i.e., random spacing of scattering objects, and movement of objects from one transmission to the next);

(j) possible multiple-scattering effects and interaction between objects are neglected;

(k) excess attenuation from power extinction caused by volume scattering is neglected; and

(l) the same transmit electrical power $\Pi_{T}$ is used for $\sigma_{b s}$ and $s_{v}$ measurements, i.e., in calibration and surveying.

These are all common assumptions underlying the traditional theory of fish abundance measurement [1-16]. The discussion of their validity is an extensive and complex subject, beyond the scope of the present work, and discussed elsewhere. Assumptions (a) and (b) relate to the transducer and electric components of the echo sounder system, and are normally fulfilled by driving the piezoelectric transducer using low electrical power. In relation to (c): in abundance estimation, the sound velocity is typically taken to be the average value of the sound velocity profile, over the depth range in question [24]. Assumption (d) is addressed by refs. [13,24-26], and maximum electrical transmission powers have been suggested [24]. Assumption (f) is discussed e.g., in ref. [27]. The assumptions $(\mathrm{g})-(\mathrm{k})$ are included in the set of assumptions used by Clay and Medwin [1,5] to derive the analogous "in-water" expressions for $s_{v}$, accounting for acoustic pressures in the sea only. Relatively extensive discussions on the validity of $(\mathrm{g})-(\mathrm{k})$ are given by refs. [1,5,6,28], also summarizing other studies addressing these issues.

A spherical coordinate system is used, with coordinates $(r, \theta, \phi)$, origin at the centre of the transducer front, and with the $z$ axis (i.e., $\theta=\phi=0$ ) chosen normal to the transducer's front surface, and assumed coincident with the transducer's acoustical beam axis [16]. $r$ is the radial distance, denoted range, $\theta$ is the polar angle (rel. to the $z$ axis), and $\phi$ is the azimuthal angle (rel. to the $x$ axis). Bold-face letters are used to indicate complex-valued quantities.

\subsection{Average Power Formulation ("Formulation $A$ ")}

Under the above assumptions, it can be shown from basic acoustic principles that the backscattering cross section of a single scattering target located at position $(r, \theta, \phi)$ in the transducer's far field, $\sigma_{b s}$, and the volume backscattering coefficient for a thin spherical shell "ping volume" $V_{p}$ in the far field, $s_{v}$, are given by the power budget equations $[11,13,16]$ :

$$
\sigma_{b s}=\frac{16 \pi^{2} \cdot r^{4} \cdot e^{4 \alpha r} \cdot \Pi_{R}^{s t}}{G^{2}(\theta, \phi) \cdot \lambda^{2} \cdot F_{\Pi} \cdot \Pi_{T}}
$$




$$
s_{v}=\frac{32 \pi^{2} \cdot r^{2} \cdot e^{4 \alpha r} \cdot \Pi_{R}^{v}}{G_{0}^{2} \cdot \psi \cdot \lambda^{2} c_{0} \cdot \tau_{p} \cdot F_{\Pi} \cdot \Pi_{T}}
$$

respectively. $\sigma_{b s}$ and $s_{v}$ are given in units of $\mathrm{m}^{2}$ and $\mathrm{m}^{-1}$, respectively. Here, $\tau_{p}$ is the duration of the transmitted voltage signal (denoted "pulse duration"). In Equation (1), $r$ is the distance to the single target. In Equation (2), $r \approx r_{p} \equiv\left(r_{p 1}+r_{p 2}\right) / 2$ is the mid-range of the "ping volume" $V_{p}$, that is contained within ranges $r_{p 1}$ and $r_{p 2}$, with thickness $d r_{p} \equiv r_{p 2}-r_{p 1}=\frac{1}{2} c_{0} \tau_{p}$ [16]. In practice, $\tau_{p}=1$ ms may often be used, corresponding to $d r_{p}=0.75 \mathrm{~m}$. $\alpha$ is the sound pressure acoustic absorption coefficient of seawater (expressed in $\mathrm{Np} / \mathrm{m}$ ). $\lambda=c_{0} / f$ is the acoustic wavelength, where $c_{0}$ is the small-signal sound velocity in seawater.

$G(\theta, \phi)$ and $G_{0}$ (both non-dimensional) are the transducer gain and the axial transducer gain, defined as $[11,13,16,29]$ :

$$
\begin{gathered}
G(\theta, \phi) \equiv \eta \cdot D(\theta, \phi)=G_{0} \cdot\left|\boldsymbol{B}_{i}(\theta, \phi)\right|^{2} \\
G_{0} \equiv G(0,0)=\eta \cdot D_{0},
\end{gathered}
$$

respectively, where $\eta$ is the transducer's electroacoustic conversion efficiency (the non-dimensional ratio of radiated acoustic to transmitted electrical power), and

$$
\begin{gathered}
D_{0}=\frac{4 \pi}{\int_{4 \pi}\left|\boldsymbol{B}_{i}(\theta, \phi)\right|^{2} d \Omega} \\
D(\theta, \phi)=\frac{4 \pi \cdot\left|\boldsymbol{B}_{i}(\theta, \phi)\right|^{2}}{\int_{4 \pi}\left|\boldsymbol{B}_{i}(\theta, \phi)\right|^{2} d \Omega}=D_{0} \cdot\left|\boldsymbol{B}_{i}(\theta, \phi)\right|^{2}
\end{gathered}
$$

and $\boldsymbol{B}_{i}(\theta, \phi)$, are the axial directivity factor [30], the directivity factor [29], and the beam pattern (the angular distribution of the sound pressure, normalized to the axial sound pressure) [30], respectively, for the transmitted sound pressure field.

$\psi$ (in steradians, sr) is the equivalent two-way beam solid angle of the transducer, defined by $[1,5,10,11,16,29]$ :

$$
\psi \equiv \int_{4 \pi}\left|\boldsymbol{B}_{i}(\theta, \phi)\right|^{4} d \Omega=\frac{1}{G_{0}^{2}} \int_{4 \pi} G^{2}(\theta, \phi) d \Omega .
$$

$\psi$ is normally provided by the echo sounder manufacturer, for the frequencies in question.

The average transmitted and received electrical powers at the transducer terminals $(\mathrm{W})$ are given as $[16,30]$ :

$$
\begin{gathered}
\Pi_{T}=\frac{\left|\boldsymbol{V}_{T}\right|^{2} R_{T}}{2\left|\boldsymbol{Z}_{T}\right|^{2}} \\
\Pi_{R}^{s t}=\frac{\left|\boldsymbol{V}_{R}^{s t}\right|^{2} R_{E}}{2\left|\boldsymbol{Z}_{E}\right|^{2}} \\
\Pi_{R}^{v}=\frac{\left|\boldsymbol{V}_{R}^{v}\right|^{2} R_{E}}{2\left|Z_{E}\right|^{2}}
\end{gathered}
$$

respectively, where "average" refers to averaging over one cycle of the harmonic signal waveform, at the frequency $f . \boldsymbol{V}_{T}, \boldsymbol{V}_{R}^{s t}$, and $\boldsymbol{V}_{R}^{v}$ are the spectral amplitudes of the voltage signals across the transducer's electrical terminals during transmission and reception, i.e., for the transmitted signal and the single-target and volume backscattered echoes, respectively. Subscripts " $T$ " and " $R$ " indicate "transmit" and "receive", and superscript "st" and " $v$ " indicate "single target" and "volume backscattering", respectively. For Equations (1), (2), and (8)-(10) it has been assumed that the same transmit signal $V_{T}(t)$ and integration time $\tau_{p}$ are used in single-target (e.g., calibration) and oceanic survey operations, respectively. 
$\mathbf{Z}_{T}=R_{T}+i X_{T}$ is the transducer's input electrical impedance at transmission, when radiating into the fluid, with resistance and reactance $R_{T}$ and $X_{T}$, respectively. $Z_{R}=R_{R}+i X_{R}$ is the output (internal) electrical impedance of the receiving transducer, and $Z_{E}=R_{E}+i X_{E}$ is the input electrical impedance of the receiving electric network (the transducer's "electrical termination impedance"), involving resistances and reactances $R_{R}$ and $X_{R}$, and $R_{E}$ and $X_{E}$, respectively. (The roles of these electrical impedances in electrical equivalent circuits of the transmitting and receiving parts of the measurement system are indicated in Figures 3 and 4 of ref. [16].) $Z_{T}$ and $\boldsymbol{Z}_{E}$ should normally be available from the echo sounder manufacturer. The assumption $\boldsymbol{Z}_{R}=\boldsymbol{Z}_{T}$ is often used.

Finally,

$$
F_{\Pi} \equiv \frac{4 R_{T} R_{E}}{\left|Z_{R}+Z_{E}\right|^{2}}
$$

is a (non-dimensional) electrical termination factor for the power-budget equations formulated in terms of average power $[13,16] . F_{\Pi}$ accounts for arbitrary electrical termination at signal reception.

Equations (1) and (2) are equivalent to the expressions given in the EK500 manual [11], except for the factor $F_{\Pi}$, which was neglected there, implicitly implying $F_{\Pi}=1[13,16]$, cf. Section 4.1.

Dividing $\sigma_{b s}$ with a reference area (chosen equal to $1 \mathrm{~m}^{2}$ ), multiplying $s_{v}$ by a reference length (chosen equal to $1 \mathrm{~m}$ ), and applying $10 \log$ on both sides of Equations (1) and (2), leads to logarithmic (dB) versions of Equations (1) and (2), commonly denoted as target strength, TS, and volume backscattering strength, $S_{v}$, respectively [22].

Equations (1) and (2) constitute formulation A of the power-budget equations for $\sigma_{b s}$ and $s_{v}$. Physical interpretations of Equations (1) and (2) in terms of power flow are given in [22].

\subsection{Echo Integration Formulation ("Formulation B")}

\subsubsection{Power Budget Equations}

Calculation of the electrical powers $\Pi_{T}, \Pi_{R}^{s t}$, and $\Pi_{R}^{v}$ involved in the average power formulation of the power-budget equations, Equations (1) and (2), is normally made using time integration of voltage signals, denoted echo integration [3,4,6-8]. An echo integration formulation of the power-budget equations for $\sigma_{b s}$ and $s_{v}$ (denoted "formulation B") is given in the following, derived from Equations (1) and (2).

From Equations (1), (2), and (8)-(10) one obtains

$$
\begin{gathered}
\left|\mathrm{V}_{R}^{s t}(f)\right|^{2}=\frac{\sigma_{b s}(f) \cdot G^{2}(\theta, \phi, f) \cdot \lambda^{2}(f) \cdot F_{V V}^{2}(f)}{16 \pi^{2} \cdot r^{4} \cdot e^{4 \alpha(f) r} \cdot\left|\mathrm{V}_{T}(f)\right|^{2}} \\
\left|\mathrm{~V}_{R}^{v}(f)\right|^{2}=\frac{c_{0} \tau_{p} \cdot s_{v}(f) \cdot G_{0}^{2}(f) \cdot \psi(f) \cdot \lambda^{2}(f) \cdot F_{V V}^{2}(f)}{32 \pi^{2} \cdot r^{2} \cdot e^{4 \alpha(f) r}} \cdot\left|\mathrm{V}_{T}(f)\right|^{2},
\end{gathered}
$$

where all quantities being a function of the frequency, $f$, have been indicated by the argument $(f)$, and

$$
F_{V V} \equiv \frac{2 R_{T}\left|Z_{E}\right|}{\left|Z_{R}+Z_{E}\right|\left|Z_{T}\right|}
$$

is the (non-dimensional) electrical termination factor for the power-budget equations formulated in terms of echo integration [16]. $F_{V V}$ accounts for arbitrary electrical termination at signal reception [16]. Integration over all frequencies gives

$$
\int_{-\infty}^{\infty}\left|\mathrm{V}_{R}^{s t}(f)\right|^{2} d f=\frac{1}{16 \pi^{2} \cdot r^{4}} \cdot \int_{-\infty}^{\infty} \frac{\sigma_{b s}(f) \cdot G^{2}(\theta, \phi, f) \cdot \lambda^{2}(f) \cdot F_{V V}^{2}(f)}{e^{4 \alpha(f) r}} \cdot\left|\mathrm{V}_{T}(f)\right|^{2} d f
$$




$$
\int_{-\infty}^{\infty}\left|\mathrm{V}_{R}^{v}(f)\right|^{2} d f=\frac{c_{0} \tau_{p}}{32 \pi^{2} \cdot r^{2}} \cdot \int_{-\infty}^{\infty} \frac{s_{v}(f) \cdot G_{0}^{2}(f) \cdot \psi(f) \cdot \lambda^{2}(f) \cdot F_{V V}^{2}(f)}{e^{4 \alpha(f) r}} \cdot\left|\mathrm{V}_{T}(f)\right|^{2} d f
$$

Now, assume that the transmitted signal, $V_{T}(t)$, is a narrowband signal with carrier frequency $f_{0}$ (e.g., a tone burst of duration » $1 / f_{0}$ ). The magnitude frequency spectrum $\left|\mathrm{V}_{T}(f)\right|$ then has a peak value at $f_{0}$, and a narrow main pass-band around $f_{0}$, with relatively low side band level. Consequently, the dominating contributions to the integrands of Equations (15)-(16) may be assumed to come from the frequency region around $f_{0}$. Under this assumption Equations (15)-(16) are approximately given as

$$
\begin{gathered}
\int_{-\infty}^{\infty}\left|\mathrm{V}_{R}^{s t}(f)\right|^{2} d f \approx \frac{\sigma_{b s}\left(f_{0}\right) \cdot G^{2}\left(\theta, \phi, f_{0}\right) \cdot \lambda^{2}\left(f_{0}\right) \cdot F_{V V}^{2}\left(f_{0}\right)}{16 \pi^{2} \cdot r^{4} \cdot e^{4 \alpha\left(f_{0}\right) r}} \cdot \int_{-\infty}^{\infty}\left|\mathrm{V}_{T}(f)\right|^{2} d f \\
\int_{-\infty}^{\infty}\left|\mathrm{V}_{R}^{v}(f)\right|^{2} d f \approx \frac{c_{0} \tau_{p} \cdot s_{v}\left(f_{0}\right) \cdot G_{0}^{2}\left(f_{0}\right) \cdot \psi\left(f_{0}\right) \cdot \lambda^{2}\left(f_{0}\right) \cdot F_{V V}^{2}\left(f_{0}\right)}{32 \pi^{2} \cdot r^{2} \cdot e^{4 \alpha\left(f_{0}\right) r}} \cdot \int_{-\infty}^{\infty}\left|\mathrm{V}_{T}(f)\right|^{2} d f
\end{gathered}
$$

From Parseval's theorem [31] the energy contained in a signal's frequency spectrum is equal to the energy contained in the signal itself. Consequently, integration over frequency can be expressed as integration over time, giving

$$
\begin{gathered}
\int_{-\infty}^{\infty}\left|V_{R}^{s t}(t)\right|^{2} d t \approx \frac{\sigma_{b s}\left(f_{0}\right) \cdot G^{2}\left(\theta, \phi, f_{0}\right) \cdot \lambda^{2}\left(f_{0}\right) \cdot F_{V V}^{2}\left(f_{0}\right)}{16 \pi^{2} \cdot r^{4} \cdot e^{4 \alpha\left(f_{0}\right) r}} \cdot \int_{-\infty}^{\infty}\left|V_{T}(t)\right|^{2} d t \\
\int_{-\infty}^{\infty}\left|V_{R}^{v}(t)\right|^{2} d t \approx \frac{c_{0} \tau_{p} \cdot s_{v}\left(f_{0}\right) \cdot G_{0}^{2}\left(f_{0}\right) \cdot \psi\left(f_{0}\right) \cdot \lambda^{2}\left(f_{0}\right) \cdot F_{V V}^{2}\left(f_{0}\right)}{32 \pi^{2} \cdot r^{2} \cdot e^{4 \alpha\left(f_{0}\right) r}} \cdot \int_{-\infty}^{\infty}\left|V_{T}(t)\right|^{2} d t
\end{gathered}
$$

In practice, the time integrations of Equations (19)-(20) are evaluated over finite time intervals, corresponding to the time gatings used for the transmitted and received signals.

As defined above, $\tau_{p}$ is the time duration of the transmitted signal $V_{T}(t)$, starting at $t=0$. Let $\tau_{s t} \equiv t_{s t 2}-t_{s t 1}$ be the time duration of the voltage echo $V_{R}^{s t}(t)$ that is received from a single scattering target, where $t_{s t 1}$ and $t_{s t 2}$ are the arrival times of the start and end of $V_{R}^{s t}(t)$. Similarly, let $\tau_{s p h} \equiv t_{s p h 2}-t_{s p h 1}$ be the time duration of the voltage echo $V_{R}^{s p h}(t)$ that is received from the calibration sphere, where $t_{s p h 1}$ and $t_{s p h 2}$ are the arrival times of the start and end of $V_{R}^{s p h}(t)$. Let $\tau_{g}=t_{g 2}-t_{g 1}$ be the "gate opening time" used for the voltage waveform $V_{R}^{v}(t)$ that is received from the "gated volume", $V_{g}$, where $t_{g 1}$ and $t_{g 2}$ are the times of gate opening and closure [16].

$\tau_{s p h}$ is always larger than $\tau_{p}$, due to "ringing" (transient decay) caused by limited transducer bandwidth, possible electronic filtering, the finite dimensions of the sphere, possible "ringing" due to elastic sphere vibration modes and circumferential waves at the sphere surface, etc., in the sphere echo. Calibration spheres are normally made to produce strong echoes, and for typical calibration distances in the range 10-25 m, the signal-to-noise ratio (SNR) is very high. Sphere echoes are significantly stronger than fish echoes, and possible false echoes (from fish or other objects) may be excluded from signal processing. If the presence of fish becomes a problem, the calibration exercise is normally paused. A voltage detection threshold can then be used to electronically estimate $t_{s p h 1}$ and $t_{s p h 2}$, determining $\tau_{s p h}$. In practice, a slightly different method may often be used. A time window is then set manually, starting just before $t_{s p h 1}$ and ending just after $t_{s p h 2}$, sufficiently wide to include all calibration sphere echoes from different sphere positions over the transducer's beam, in the vicinity of the acoustic axis. Which method to use is a question of uncertainty.

With respect to $t_{s t}$, the time duration of single-target echoes (e.g., individual fish), a fish produces a weaker echo than a calibration sphere, so for the same measurement distance, the SNR is lower. The 
voltage detection threshold method can still be used in most cases, determining $t_{s t 1}$ and $t_{s t 2}$ to give $\tau_{s t}$. One has to ensure that possible false echoes (from other fish or objects) are excluded from processing of the signal.

$\tau_{g}$ may be smaller, equal to, or larger than $\tau_{p}$, depending on the echo integration solutions implemented in the echo sounder system $[1,5,16]$. A choice $\tau_{g} \geqslant \tau_{p}$ may often be relevant in practice. The operator of the instrument typically sets the "channel depth" (equal to $\frac{1}{2} c_{0} \tau_{g}$ ) of the "gated volume" $\left(V_{g}\right) .10 \mathrm{~m}$ may be common in open sea (corresponding to $\tau_{g} \approx 133 \mathrm{~ms}$ ), and 1-2 $\mathrm{m}$ close to the sea bottom (corresponding to $\tau_{g} \approx 13-27 \mathrm{~ms}$ ). $\tau_{g}$ depends on the measured sound velocity, $c_{0}$. In general one thus has $\tau_{p} \neq \tau_{s t} \neq \tau_{s p h} \neq \tau_{g}$.

Consequently, due to different time gatings of the different signals, one cannot compare the signal energies directly, since these depend on the integration times. However, the time averaged energies can be compared, i.e., the effective (rms) values of the time gated signals. Equations (19)-(20) may thus be approximated by

$$
\begin{gathered}
\frac{1}{\tau_{s t}} \int_{t_{s t 1}}^{t_{s t 1}}\left|V_{R}^{s t}(t)\right|^{2} d t \approx \frac{\sigma_{b s}\left(f_{0}\right) \cdot G^{2}\left(\theta, \phi, f_{0}\right) \cdot \lambda^{2}\left(f_{0}\right) \cdot F_{V V}^{2}\left(f_{0}\right)}{16 \pi^{2} \cdot r^{4} \cdot e^{4 \alpha\left(f_{0}\right) r}} \cdot \frac{1}{\tau_{p}} \int_{0}^{\tau_{p}}\left|V_{T}(t)\right|^{2} d t \\
\frac{1}{\tau_{g}} \int_{t_{g 1}}^{t_{g 2}}\left|V_{R}^{v}(t)\right|^{2} d t \approx \frac{c_{0} \tau_{p} \cdot s_{v}\left(f_{0}\right) \cdot G_{0}^{2}\left(f_{0}\right) \cdot \psi\left(f_{0}\right) \cdot \lambda^{2}\left(f_{0}\right) \cdot F_{V V}^{2}\left(f_{0}\right)}{32 \pi^{2} \cdot r^{2} \cdot e^{4 \alpha\left(f_{0}\right) r}} \cdot \frac{1}{\tau_{p}} \int_{0}^{\tau_{p}}\left|V_{T}(t)\right|^{2} d t,
\end{gathered}
$$

giving

$$
\begin{aligned}
\sigma_{b s} & =\frac{16 \pi^{2} \cdot r^{4} \cdot e^{4 \alpha r} \cdot \tau_{p} \cdot[t i v s]_{R}^{s t}}{G^{2}(\theta, \phi) \cdot \lambda^{2} \cdot \tau_{s t} \cdot F_{V V}^{2} \cdot[t i v s]_{T}} \\
s_{v} & =\frac{32 \pi^{2} \cdot r^{2} \cdot e^{4 \alpha r} \cdot[t i v s]_{R}^{v}}{G_{0}^{2} \cdot \psi \cdot \lambda^{2} c_{0} \cdot \tau_{g} \cdot F_{V V}^{2} \cdot[t i v s]_{T}}
\end{aligned}
$$

where $\sigma_{b s}, s_{v}, \alpha, \lambda, G_{0}, G(\theta, \phi), \psi$, and $F_{V V}$ are all evaluated at the carrier frequency, $f_{0}$, and

$$
\begin{gathered}
{[t i v s]_{T} \equiv \int_{0}^{\tau_{p}}\left|V_{T}(t)\right|^{2} d t} \\
{[t i v s]_{R}^{s t} \equiv \int_{t_{s t 1}}^{t_{s t 2}}\left|V_{R}^{s t}(t)\right|^{2} d t} \\
{[t i v s]_{R}^{s p h} \equiv \int_{t_{s p h 1}}^{t_{s p h 2}}\left|V_{R}^{s p h}(t)\right|^{2} d t} \\
{[t i v s]_{R}^{v} \equiv \int_{t_{g 1}}^{t_{g 2}}\left|V_{R}^{v}(t)\right|^{2} d t}
\end{gathered}
$$

are defined as the "echo integral"—or "time-integral-voltage-squared" [tivs]—values of the transmitted voltage signal (the "pulse"), $V_{T}(t)$, and three different received voltage waveforms, $V_{R}^{\text {st }}(t), V_{R}^{\text {sph }}(t)$, and $V_{R}^{v}(t)$, for a single target echo (e.g., individual fish), a calibration sphere echo, and the echo received from a "gated volume" in oceanic surveying, respectively [16]. In this method, by using the effective $(\mathrm{rms})$ values of the time gated signals, $\sigma_{b s}$ and $s_{v}$ become essentially independent of pulse duration. 
By using the same transmit pulse $V_{T}(t)$ in calibration and field operation, $s_{v}$ becomes essentially independent of pulse shape, under the assumptions (b) and (d) of small-amplitude sound propagation.

The definition of the echo integrals corresponds to that given by $[3,4,6]$. The [tivs] notation used here represents a generalization of the [tips] ("time-integral-pressure-squared") notation used by Medwin and Clay [5], to account for voltage instead of "in-water" sound pressure signals [16]. The echo integrals ([tivs]) represent energy times electrical impedance, in units of $\mathrm{V}^{2} \mathrm{~s}=\mathrm{W} \cdot \mathrm{ohm} \cdot \mathrm{s}=\mathrm{J} \cdot \mathrm{ohm}$.

\subsubsection{Formulation B Calibration Factor $G_{0}$}

Now, consider calibration of the echo sounder or sonar system using a single metal sphere, with a theoretically known value for the backscattering cross section of the sphere, $\sigma_{b s, \text { theory }}^{s p h}$ calculated at the frequency $f_{0}$ in question [or the corresponding target strength $T S_{\text {theory }}^{\text {sph }} \equiv 10 \log \left(\sigma_{b s, \text { theory }}^{s p h} / r_{1}^{2}\right)$, for a reference area $r_{1}^{2}=1 \mathrm{~m}^{2}$ ]. Consider a calibration situation with the centre of the calibration sphere located at position $\left(r_{s p h}, \theta_{s p h}, \phi_{s p h}\right)$. (Quantities and parameters that are measured or assumed known at calibration using the metal calibration sphere, are given sub- or superscript "sph".)

Formulation B involves use of a single calibration fator, $G_{0}$. Two approaches are used to derive $G_{0}$ expressions for use in calibration. The first approach (denoted "Method 1") gives $G_{0}$ directly in terms of the basic quantities being measured in calibration. The second approach (denoted "Method 2") is used to establish an alternative relationship for use in calibration that is similar to those used in [11] for the two calibration factors of the EK500 system, cf. Equations (47) and (50).

The basic quantities being measured in echo sounder calibration are $r_{s p h}, \theta_{s p h}, \phi_{s p h}, \tau_{p}, \tau_{s p h}$, $[t i v s]_{R}^{s p h},[t i v s]_{T},\left|\boldsymbol{B}_{i}\left(\theta_{s p h}, \phi_{s p h}\right)\right|$ and $c_{0, s p h} . F_{V V, s p h}$ and $\sigma_{b s, \text { theory }}^{\text {sph }}$ are typically assumed to be known. These quantities are all evaluated at the carrier frequency of the transmitted signal, $f_{0}$.

Method 1:

From Equations (3), (4), and (23), $G_{0}$ can be determined from calibration measurements as

$$
G_{0}^{2}=\frac{16 \pi^{2} \cdot r_{s p h}^{4} \cdot e^{4 \alpha r_{s p h}} \cdot \tau_{p} \cdot[t i v s]_{R}^{s p h}}{\lambda_{s p h}^{2} \cdot F_{V V, s p h}^{2} \cdot \tau_{s p h} \cdot \sigma_{b s, t h e o r y}^{s p h} \cdot\left|B_{i}\left(\theta_{s p h}, \phi_{s p h}\right)\right|^{4} \cdot[t i v s]_{T}} .
$$

Method 2:

An alternative and equivalent method to determine $G_{0}$ by echo sounder calibration using the metal sphere, may be derived as follows. From Equations (3), (4), and (23) one has

$$
G_{0}^{2} \cdot \sigma_{b s}^{s p h}=\frac{16 \pi^{2} \cdot r_{s p h}^{4} \cdot e^{4 \alpha r_{s p h}} \cdot \tau_{p} \cdot[t i v s]_{R}^{s p h}}{\lambda_{s p h}^{2} \cdot F_{V V, s p h}^{2} \cdot \tau_{s p h} \cdot\left|B_{i}\left(\theta_{s p h}, \phi_{s p h}\right)\right|^{4} \cdot[t i v s]_{T}} \equiv C_{1},
$$

where $C_{1}$ is a constant and known (measured) value for a given calibration measurement. Therefore, by knowing $\sigma_{b s, t h e o r y}^{s p h}$ the relevant calibration value $G_{0}$ is given by $G_{0}^{2}=C_{1} / \sigma_{b s, t h e o r y}^{s p h}$.

Consider a situation where an old (or an arbitrary, and possibly erroneous) calibration value is used initially in the calibration measurement, denoted $G_{0, \text { old }}$. The corresponding value measured initially for the backscattering coefficient of the calibration sphere is then $\sigma_{b s, o l d}^{s p h}=C_{1} / G_{0, \text { old }}^{2}$. It follows that $C_{1}=G_{0}^{2} \cdot \sigma_{b s, \text { theory }}^{s p h}=G_{0, o l d}^{2} \cdot \sigma_{b s, o l d}^{s p h}$ giving

$$
\begin{gathered}
G_{0}^{2}=G_{0, \text { old }}^{2} \cdot \frac{\sigma_{b s, \text { old }}^{\text {sph }}}{\sigma_{b s, \text { theory }}^{\text {sph }}} \\
10 \log \left(G_{0}\right)=10 \log \left(G_{0, \text { old }}\right)+\frac{1}{2} \cdot\left[T S_{\text {old }}^{\text {sph }}-T S_{\text {theory }}^{\text {sph }}\right] .
\end{gathered}
$$


(Since from instrument manuals and relevant literature (cf. e.g., [11,18-21]) the calibration factors of modern scientific echo sounder and sonar systems are essentially given on logarithmic form, many users of such equipment know these factors in logarithmic form only. For completeness and convenience, both the linear and logarithmic expressions for the calibration factors are thus given here and in Section 3.)

The calibration value $G_{0}$ may thus be calculated from the old calibration value, $G_{0, o l d} . \sigma_{b s, o l d}^{s p h}$ corresponds to the target strength $T S_{\text {old }}^{s p h} \equiv 10 \log \left(\sigma_{b s, o l d}^{s p h} / r_{1}^{2}\right)[22]$.

From these equations it follows that Equations (31) and (32) are equivalent to Equation (29). It thus follows that Methods 1 and 2 for determination of $G_{0}$ under Formulation B, are both generally valid approaches, leading to the same calibration value for $G_{0}$.

Equations (23) and (24) for $\sigma_{b s}$ and $s_{v}$, together with Equation (29) or Equation (31) for the calibration factor $G_{0}$, constitute formulation $B$ of the functional relationship for fish abundance estimation.

\subsection{3. "Compact Formulation B" Functional Relationship}

A compact version of Formulation B may be derived as follows. Insertion of Equation (29) into Equations (23) and (24) yields

$$
\begin{aligned}
& \sigma_{b s}=e^{4 \alpha\left(r-r_{s p h}\right)} \cdot\left(\frac{r}{r_{s p h}}\right)^{4} \cdot\left(\frac{\lambda_{s p h}}{\lambda}\right)^{2} \cdot\left(\frac{F_{V V, s p h}}{F_{V V}}\right)^{2} \cdot \frac{\tau_{s p h}}{\tau_{s t}} \cdot \frac{[t i v s]_{R}^{s t}}{[t i v s]_{R}^{s p h}} \cdot\left|\frac{\boldsymbol{B}_{i}\left(\theta_{s p h}, \phi_{s p h}\right)}{\boldsymbol{B}_{i}(\theta, \phi)}\right|^{4} \cdot \sigma_{b s, \text { theory }}^{s p h} \\
& s_{v}=e^{4 \alpha\left(r-r_{s p h}\right)} \cdot \frac{r^{2}}{r_{s p h}^{4}} \cdot\left(\frac{\lambda_{s p h}}{\lambda}\right)^{2} \cdot\left(\frac{F_{V V, s p h}}{F_{V V}}\right)^{2} \cdot \frac{2}{c_{0} \tau_{g}} \cdot \frac{\tau_{s p h}}{\tau_{p}} \cdot \frac{[t i v s]_{R}^{v}}{[t i v s]_{R}^{s p h}} \cdot \frac{\left|\boldsymbol{B}_{i}\left(\theta_{s p h}, \phi_{s p h}\right)\right|^{4}}{\psi} \cdot \sigma_{b s, t h e o r y}^{s p h}
\end{aligned}
$$

respectively. Equations (33)-(34) represent an alternative and equivalent generic echo integration functional relationship for fish abundance estimation, given in terms of the basic quantities being measured during calibration and oceanic surveying [22].

Equations (33)-(34) reveal which parameters that influence the calibration and oceanic survey measurements. These expressions may be particularly useful for uncertainty or sensitivity studies, such as with respect to effects of sea temperature, or other possible errors or drift in the parameters involved in abundance measurement and species identification. The influence on the measurement accuracy from an uncertainty-or a possible error-in each of the quantities being measured or assumed known in calibration and oceanic survey operation can be investigated by adding an uncertainty term for this (or these) parameter(s), and using Equations (33)-(34) to calculate the resulting changes in $\sigma_{b s}$ and $s_{v}$.

\subsubsection{Fish Density Estimation}

$s_{\mathrm{V}}$ as given by Equation (24), or equivalently, Equation (34), represents volume backscattering from the thin spherical shell sub-volume $V_{p}$ of thickness $d r_{p}=\frac{1}{2} c_{0} \tau_{p}$ in the observation volume $V_{o b s}$. The volume backscattering from $V_{o b s}$, between ranges $r_{\min }$ and $r_{\max }$, is obtained by measuring $s_{\mathrm{V}}$ for a continuous sequence of "ping volumes", $V_{p}$, and integrating $s_{\mathrm{V}}$ over the range of these volumes, giving the area backscattering coefficient of $V_{o b s}[11,16,17]$ :

$$
s_{a} \equiv \int_{r_{\min }}^{r_{\max }} s_{v}(r) d r,
$$


representing the backscattering cross section per unit area, within $V_{o b s}$. Due to the integration of $s_{v}$ (in uints of $\mathrm{m}^{-1}$ ) over distance, $s_{a}$ is a non-dimensional quantity. In echo sounder output, $s_{a}$ is frequently given in terms of the nautical area scattering coefficient (NASC) $[6,11,17]$ :

$$
s_{A} \equiv 4 \pi \cdot 1852^{2} \cdot s_{a}
$$

where $s_{a}$ has been multiplied by the surface area of a sphere with radius one nautical mile. The density of targets (fish), expressed as the (non-dimensional) number of specimens in $V_{o b s}$ per square nautical mile, is then given as [17]

$$
\rho_{a}=\frac{s_{A}}{4 \pi\left\langle\sigma_{b s}\right\rangle}
$$

where $\left\langle\sigma_{b s}\right\rangle$ is the expected value of the backscattering cross section (representing the expected TS) of individual targets (fish) [3,6,8,10]. Insertion of Equation (24) in Equations (35)-(37) yields [16]

$$
\rho_{a}=\frac{C_{c a l}}{\psi\left\langle\sigma_{b s}\right\rangle} E
$$

where

$$
\begin{gathered}
E \equiv \int_{r_{\min }}^{r_{\max }} r^{2} e^{4 \alpha r}[t i v s]_{R}^{v} d r \\
C_{c a l} \equiv \frac{32 \pi^{2} \cdot 1852^{2}}{G_{0}^{2} \cdot \lambda^{2} c_{0} \cdot \tau_{g} \cdot F_{V V}^{2} \cdot[t i v s]_{T}} .
\end{gathered}
$$

Equation (38) is in the form of the traditional echo-integrator equation $[3,4,6,8,10,16]$, where $C_{c a l}$ represents the calibration factor used in that equation. $E$ is a "range integrated echo integral" for the observation volume $V_{o b s}$, obtained by integrating the sequence of echo integrals [tivs $]_{R}^{v}$, each associated with a "ping volume" $V_{p}$. For each $V_{p}$ at range $r$ in $V_{o b s}$, the term $r^{2} e^{4 \alpha r}$ in $E$ is the usual "20log $(r)+2 \hat{\alpha} r$ " TVG (time-varied gain) factor for volume backscattering from $V_{p}$ [3], where $\hat{\alpha}$ is the absorption coefficient expressed in $\mathrm{dB} / \mathrm{m}$. Similarly, for each $V_{p}$, $[t i v s]_{R}^{v}$ is the echo integral for the "gated volume" $V_{g}$, taken over the time interval $\tau_{g}$, and typically averaged over many transmissions [3,4]. Since [tivs $]_{R}^{v}$ is calculated for each $V_{p}$, it depends on range, $r$. In refs. $[3,4,6,10] E$ is referred to as the "echo-integrator output".

Equations (38)-(40) give the connection between the theory presented here (Formulations A-E), and the traditional echo-integrator equation used e.g., by $[3,4,6,8,10]$. In that literature, $C_{c a l}$ was used solely as an unspecified calibration factor. Here, the functional relationship for $C_{c a l}$ is derived, and fully given for small-amplitude conditions in terms of the echo sounder system parameters.

\section{Application to Echo Sounder and Sonar Systems}

For single-target measurements (such as in calibration, or for TS measurement of individual fish), some manufacturers base their $\sigma_{b s}$ (i.e., TS) measurement on the peak value of the echo samples in the single target echo, and not the echo signal waveform itself, as used for $s_{v}$ measurements in survey operation [11,18-21]. Cf. Equations (25)-(28). In the following the consequences of such a "peak voltage echo integration" approach in single-target TS measurements are investigated.

For this specific signal processing approach, the generic theory of Section 2 (Formulation B) is used to derive three alternative formulations of the power-budget equations with calibration factors, denoted "Formulation C", "D", and "E", respectively. Since-for these three formulations-a specific signal processing method is employed for integrating the calibration sphere echo, Formulations $C-E$ are less generic than Formulation B, but still equivalent to Formulation B, and fully valid for this approach.

The details of the mathematical derivations are given in [22], and omitted here. Only the resulting expressions for Formulations C-E are summarized, as a basis for the discussion of Section 4. In a 
few cases equations are duplicated in Section 3. This is done for completeness and convenience in summarizing each of Formulations C-E.

\subsection{Echo Integration "Formulation C"}

\subsubsection{Power-Budget Equations}

Using the approach described above with "peak voltage echo integration" in single-target TS measurements, $\sigma_{b s}$ and $s_{v}$ given by Equations (23) and (24) can equivalently be expressed as [22]:

$$
\begin{gathered}
\sigma_{b s}=\frac{16 \pi^{2} \cdot r^{4} \cdot e^{4 \alpha r} \cdot[\text { tivs }]_{R, \max }^{s t}}{G_{e f f}^{2}(\theta, \phi) \cdot \lambda^{2} \cdot F_{V V}^{2} \cdot[t i v s]_{T}} \\
s_{v}=\frac{32 \pi^{2} \cdot r^{2} \cdot e^{4 \alpha r} \cdot[\text { tivs }]_{R}^{v}}{G_{0}^{2} \cdot \psi \cdot \lambda^{2} c_{0} \cdot \tau_{g} \cdot F_{V V}^{2} \cdot[\text { tivs }]_{T}},
\end{gathered}
$$

where

$$
\begin{gathered}
{[t i v s]_{R, \max }^{s t} \equiv\left|V_{R}^{s t}\right|_{\max }^{2} \cdot \tau_{p}} \\
G_{e f f}^{2}(\theta, \phi) \equiv G^{2}(\theta, \phi) \cdot \frac{\tau_{s t} \cdot\left|V_{R}^{s t}\right|_{\max }^{2}}{[t i v s]_{R}^{s t}}
\end{gathered}
$$

Here [22], [tivs $]_{R, \max }^{s t}$ is a simplified (and erroneous) estimate of the single target echo integral $[t i v s]_{R}^{s t}$, obtained by multiplying the transmit signal duration $\tau_{p}$ with the constant squared voltage value, $\left|V_{R}^{s t}\right|_{\max }^{2} \cdot\left|V_{R}^{s t}\right|_{\max }$ is the maximum ("peak voltage") value of the magnitude of the single target echo, $\left|V_{R}^{s t}(t)\right|$, within the time window $\left[t_{s t 1}, t_{s t 2}\right]$. $G_{e f f}^{2}(\theta, \phi)$ represents an "effective" value of $G^{2}(\theta, \phi)$, where a correction factor has been included to compensate for the error introduced by using $[\text { tivs }]_{R, \max }^{s t}$ instead of $[t i v s]_{R}^{s t}$, to ensure that the resulting $\sigma_{b s}$ measurement is valid and correct [22].

This approach represents a "rectangular echo integration" in single-target TS measurements, i.e., for $\sigma_{b s}$. For $s_{v}$, full waveform echo integration is used, as in Formulation B, and Equation (42) is identical to Equation (24).

\subsubsection{Formulation C Calibration Factors $G_{0, e f f}$ and $G_{0}$}

Formulation $C$ involves two calibration factors, $G_{0, e f f}$ and $G_{0}$, where $G_{0, e f f} \equiv G_{\text {eff }}(0,0)$. Two approaches are used to derive expressions for these. The first approach (denoted "Method 1") gives $G_{0, e f f}$ and $G_{0}$ in terms of the basic quantities being measured in calibration. The second approach (denoted "Method 2") is used to (i) establish alternative relationships for use in calibration; and (ii) establish relationships for the two Formulation D calibration factors, $G_{0, e f f}$ and $G_{0}$, that can be used for comparison with the two EK500 calibration factors "TS transducer gain" and " $S_{v}$ transducer gain" (cf. Section 4.3).

The calibration factor $G_{0, e f f}$ can be expressed by two equivalent expressions [22]:

Method 1:

$$
G_{0, e f f}^{2} \equiv \frac{16 \pi^{2} \cdot r_{s p h}^{4} \cdot e^{4 \alpha r_{s p h}} \cdot[t i v s]_{R, \max }^{s p h}}{\lambda_{s p h}^{2} \cdot \sigma_{b s, t h e o r y}^{s p h} \cdot\left|\boldsymbol{B}_{i}\left(\theta_{s p h}, \phi_{s p h}\right)\right|^{4} \cdot F_{V V, s p h}^{2} \cdot[t i v s]_{T}}
$$

Method 2:

$$
\begin{gathered}
G_{0, e f f}^{2}=G_{0, e f f, \text { old }}^{2} \cdot \frac{\sigma_{b s, o l d}^{s p h}}{\sigma_{b s, \text { theory }}^{s p h}} \\
10 \log \left(G_{0, e f f}\right)=10 \log \left(G_{0, e f f, \text { old }}\right)+\frac{1}{2} \cdot\left[T S_{\text {eff } \text { old }}^{\text {sph }}-T S_{\text {theory }}^{\text {sph }}\right] .
\end{gathered}
$$


Here, $G_{0, e f f, o l d}$ is an old (or arbitrary, and possibly erroneous) calibration value used for $G_{0, e f f}$ initially in the calibration measurement. $\sigma_{b s, o l d}^{s p h}$ is the corresponding old measured value used initially for the backscattering cross section of the calibration sphere, corresponding to target strength $T S_{\text {eff } \text {,old }}^{\text {sph }} \equiv 10 \log \left(\sigma_{b s, o l d}^{s p h} / r_{1}^{2}\right)[22]$.

Similarly, the calibration factor $G_{0}$ can be expressed by two equivalent expressions [22]:

Method 1:

$$
G_{0}^{2}=\frac{16 \pi^{2} \cdot r_{s p h}^{4} \cdot e^{4 \alpha r_{s p h}} \cdot \tau_{p} \cdot[t i v s]_{R}^{s p h}}{\lambda_{s p h}^{2} \cdot F_{V V, s p h}^{2} \cdot \tau_{s p h} \cdot \sigma_{b s, t h e o r y}^{s p h} \cdot\left|B_{i}\left(\theta_{s p h}, \phi_{s p h}\right)\right|^{4} \cdot[t i v s]_{T}} .
$$

Method 2:

$$
\begin{gathered}
G_{0}^{2}=G_{0, \text { old }}^{2} \cdot \frac{s_{a, \text { old }}^{\text {sph }}}{s_{a, \text { theory }}^{\text {sph }}} \\
10 \log \left(G_{0}\right)=10 \log \left(G_{0, \text { old }}\right)+\frac{1}{2} \cdot 10 \log \left(\frac{s_{a, \text { old }}^{\text {sph }}}{s_{a, \text { theory }}^{\text {sph }}}\right),
\end{gathered}
$$

where

$$
s_{a, \text { theory }}^{s p h}=\frac{\tau_{s p h}}{\tau_{p}} \cdot \frac{\left|\boldsymbol{B}_{i}\left(\theta_{s p h}, \phi_{s p h}\right)\right|^{4}}{\psi \cdot r_{s p h}^{2}} \cdot \sigma_{b s, t h e o r y}^{s p h} .
$$

Here, $G_{0, o l d}$ is an old (or arbitrary, and possibly erroneous) calibration value used for $G_{0}$ initially in the calibration measurement. $s_{a, \mathrm{old}}^{s p h}$ is the corresponding old measured value used initially for the area backscattering coefficient of the sphere [22]. $s_{a, t h e o r y}^{s p h}$ is a known value of the area backscattering coefficient of the sphere, given from calibration measurements and the known (calculated) value $\sigma_{b s, t h e o r y}^{s p h}$. (A similar expression as Equation (51) is given in [11], but without $\left|\boldsymbol{B}_{i}\left(\theta_{\text {sph }}, \phi_{\text {sph }}\right)\right|$ and the factor $\tau_{s p h} / \tau_{p}$. That expression thus relates to the special case of calibration sphere located on the acoustic axis, $\theta_{s p h}=\phi_{s p h}=0$, and $\tau_{s p h}=\tau_{p}$ (where the latter condition may not be valid in practice).)

Equations (41) and (42) for $\sigma_{b s}$ and $s_{v}$, together with Equations (45)-(51) for the calibration factors $G_{0, e f f}$ and $G_{0}$, constitute Formulation $C$ of the functional relationship for fish abundance estimation.

\subsection{Echo Integration "Formulation D"}

\subsubsection{Power-Budget Equations}

By defining $s_{a, c o r r} \equiv G_{0} / G_{0, e f f}, \sigma_{b s}$ and $s_{v}$ given by Equations (41) and (42) can equivalently be expressed as [22]:

$$
\begin{gathered}
\sigma_{b s}=\frac{16 \pi^{2} \cdot r^{4} \cdot e^{4 \alpha r} \cdot[t i v s]_{R, \max }^{s t}}{G_{e f f}^{2}(\theta, \phi) \cdot \lambda^{2} \cdot F_{V V}^{2} \cdot[t i v s]_{T}} \\
s_{v}=\frac{32 \pi^{2} \cdot r^{2} \cdot e^{4 \alpha r} \cdot[t i v s]_{R}^{v}}{G_{0, e f f}^{2} \cdot \psi \cdot \lambda^{2} c_{0} \cdot \tau_{g} \cdot s_{a, c o r r}^{2} \cdot F_{V V}^{2} \cdot[t i v s]_{T}},
\end{gathered}
$$

where Equation (52) is identical to Equation (41), and [22]:

$$
s_{a, \text { corr }}^{2} \equiv \frac{G_{0}}{G_{0, e f f}}=\frac{[t i v s]_{R}^{s t}}{\tau_{s t} \cdot\left|V_{R}^{s t}\right|_{\max }^{2}}=\frac{\tau_{p}}{\tau_{s t}} \cdot \frac{[t i v s]_{R}^{s t}}{[t i v s]_{R, \max }^{s t}} .
$$


From Equations (9) and (26) it follows that

$$
\begin{gathered}
\Pi_{R}^{s t}=\frac{R_{E}}{\left|\boldsymbol{Z}_{E}\right|^{2}}\left(V_{R}^{s t, r m s}\right)^{2}=\frac{R_{E}}{\left|\mathbf{Z}_{E}\right|^{2}} \frac{1}{\tau_{s t}} \int_{t_{s t 1}}^{t_{s t 2}}\left|V_{R}^{s t}(t)\right|^{2} d t=\frac{R_{E}}{\left|\mathbf{Z}_{E}\right|^{2}} \frac{[t i v s]_{R}^{s t}}{\tau_{s t}} \\
\Pi_{R, \text { max }}^{s t}=\frac{R_{E}}{\left|\mathbf{Z}_{E}\right|^{2}}\left|V_{R}^{s t}\right|_{\text {max }}^{2}
\end{gathered}
$$

giving, from Equations (43) and (54),

$$
s_{a, \text { corr }}^{2}=\frac{\Pi_{R}^{s t}}{\prod_{R, \max }^{s t}} .
$$

Here, $V_{R}^{\text {st,rms }}$ is the effective (rms) amplitude of the echo received from a single target (e.g., a fish, or a calibration sphere), and $\Pi_{R, \text { max }}^{s t}$ is the "maximum average electrical power" of that echo. Equation (57) shows that $s_{a, c o r r}^{2}$ represents the (non-dimensional) ratio of the actual average electrical power, $\Pi_{R}^{s t}$, in a single-target echo (i.e., from a calibration sphere, or a fish), to the "maximum average electrical power" of that echo, $\Pi_{R, \max }^{s t}$ (i.e., the average power obtained by using "peak voltage echo integration", instead of full waveform echo integration).

\subsubsection{Formulation D Calibration Factors $G_{0, e f f}$ and $s_{a, c o r r}$}

Formulation D involves two calibration factors, $G_{0, e f f}$ and $s_{a, c o r r}$. Similarly to Formulation $C$, two approaches are used to derive expressions for these. The first approach (denoted "Method 1") gives $G_{0, e f f}$ and $s_{a, c o r r}$ in terms of the basic quantities being measured in calibration. The second approach (denoted "Method 2") is used to (i) establish alternative relationships for use in calibration; and (ii) establish relationships for the two formulation D calibration factors, $G_{0, e f f}$ and $s_{a, c o r r}$, that can be used for comparison with the two EK60 calibration factors "Gain" and "S $S_{a}$ correction" (cf. Section 4.4). For $s_{a, c o r r}$, a third approach (denoted "Method 3") is also used, which may be of interest if an EK60 type echo sounder (or similar) is operated as an EK500 type echo sounder (i.e., using calibration factors $G_{0, e f f}$ and $G_{0}$ ).

The calibration factor $G_{0, e f f}$ can be expressed by two equivalent expressions, given by Equations (45) and (46) [22].

The calibration factor $s_{a, c o r r}$ can be expressed by three equivalent expressions [22]:

Method 1:

$$
s_{a, \text { corr }}^{2}=\frac{16 \pi^{2} \cdot r_{s p h}^{4} \cdot e^{4 \alpha r_{s p h}} \cdot \tau_{p} \cdot[t i v s]_{R}^{s p h}}{G_{0, e f f}^{2} \cdot \lambda_{s p h}^{2} \cdot \tau_{s p h} \cdot \sigma_{b s, t h e o r y}^{s p h} \cdot\left|B_{i}\left(\theta_{s p h}, \phi_{s p h}\right)\right|^{4} \cdot F_{V V, s p h}^{2} \cdot[t i v s]_{T}} .
$$

Method 2:

$$
\begin{gathered}
s_{a, \text { corr }}^{2}=s_{a, \text { corr old }}^{2} \cdot \frac{s_{a, \text { old }}^{s p h}}{s_{a, \text { theory }}^{s h}} \\
S_{a, \text { corr }}=S_{a, \text { corr }, \text { old }}+\frac{1}{2} \cdot 10 \log \left(\frac{s_{a, o l d}^{s p h}}{s_{a, \text { theory }}^{s p h}}\right) .
\end{gathered}
$$

Method 3:

$$
\begin{gathered}
s_{a, \text { corr }}=\frac{G_{0}}{G_{0, e f f}} \\
S_{a, \text { corr }}=10 \log \left(G_{0}\right)-10 \log \left(G_{0, e f f}\right) .
\end{gathered}
$$


Equations (52) and (53) for $\sigma_{b s}$ and $s_{v}$, together with Equations (45)-(47) and (58)-(62), respectively, for the calibration factors $G_{0, e f f}$ and $s_{a, c o r r}$, constitute Formulation D of the functional relationship for fish abundance estimation.

\subsection{Echo Integration "Formulation E"}

\subsubsection{Power-Budget Equations}

By replacing $\tau_{g} \cdot s_{a, \text { corr }}^{2}$ with $\tau_{e f f}$ in the $s_{v}$ equation, $\sigma_{b s}$ and $s_{v}$ given by Equations (52) and (53) can equivalently be expressed as [22]:

$$
\begin{gathered}
\sigma_{b s}=\frac{16 \pi^{2} \cdot r^{4} \cdot e^{4 \alpha r} \cdot[t i v s]_{R, \max }^{s t}}{G_{e f f}^{2}(\theta, \phi) \cdot \lambda^{2} \cdot F_{V V}^{2} \cdot[t i v s]_{T}} \\
s_{v}=\frac{32 \pi^{2} \cdot r^{2} \cdot e^{4 \alpha r} \cdot[t i v s]_{R}^{v}}{G_{0, e f f}^{2} \cdot \psi \cdot \lambda^{2} c_{0} \cdot \tau_{e f f} \cdot F_{V V}^{2} \cdot[t i v s]_{T}},
\end{gathered}
$$

where Equation (63) is identical to Equations (41) and (52). From Equations (54) and (57) it follows that [22]

$$
\tau_{e f f} \equiv \tau_{g} \cdot s_{a, c o r r}^{2}=\tau_{g} \cdot \frac{\tau_{p}}{\tau_{s t}} \cdot \frac{[t i v s]_{R}^{s t}}{[t i v s]_{R, \max }^{s t}}=\tau_{g} \cdot \frac{\Pi_{R}^{s t}}{\prod_{R, \max }^{s t}} .
$$

The latter expression in Equation (65) shows that $\tau_{\text {eff }}$ represents a scaled gate opening time, where $\tau_{g}$ (used for volume backscattering in oceanic surveying) is scaled by the ratio of the actual average electrical power, $\Pi_{R}^{s t}$, to the "maximum average electrical power", $\Pi_{R, \text { max }}^{s t}$, of the single target echo. $\tau_{\text {eff }}$ may thus be interpreted as an "effective echo integration time" [22].

From Equations (26), (27), (43), and (65) it also follows that

$$
\begin{gathered}
{[t i v s]_{R}^{s t} \equiv \int_{t_{s t 1}}^{t_{s t 2}}\left|V_{R}^{s t}(t)\right|^{2} d t=\tau_{e f f} \frac{\tau_{s t}}{\tau_{g}}\left|V_{R}^{s t}\right|_{\max }^{2}} \\
{[\text { tivs }]_{R}^{s p h} \equiv \int_{t_{s p h 1}}^{t_{s p h h}}\left|V_{R}^{s p h}(t)\right|^{2} d t=\tau_{e f f} \frac{\tau_{s p h}}{\tau_{g}}\left|V_{R}^{s p h}\right|_{\max }^{2}}
\end{gathered}
$$

for the echo integrals of a single target and a calibration sphere, respectively. Consequently, for single targets, full waveform echo integration can be carried out using $\left|V_{R}^{s t}\right|_{\max }$ and $\tau_{\text {eff }}$, provided $\tau_{s t}$ and $\tau_{g}$ are known.

\subsubsection{Formulation E Calibration Factors $G_{0, e f f}$ and $\tau_{e f f}$}

Formulation E involves two calibration factors, $G_{0, e f f}$ and $\tau_{\text {eff }}$. Similarly to Formulations $C$ and $\mathrm{D}$, two approaches are used to derive expressions for these. The first approach (denoted "Method 1") gives $G_{0, e f f}$ and $\tau_{e f f}$ in terms of the basic quantities being measured in calibration. The second approach (denoted "Method 2") is used to establish alternative relationships for use in calibration. For $\tau_{\text {eff }}$, a third approach (denoted "Method 3") is also given, which may be of interest if an EK60 type echo sounder (or similar) is operated as an EK500 type of echo sounder (i.e., using calibration factors $G_{0, e f f}$ and $G_{0}$ ).

As for Formulations $C$ and $D$, the calibration factor $G_{0, e f f}$ can be expressed by two equivalent expressions, given by Equations (45) and (46) [22].

The calibration factor $\tau_{\text {eff }}$ can be expressed by three equivalent expressions [22]: 
Method 1:

$$
\tau_{\text {eff }}=\frac{16 \pi^{2} \cdot r_{s p h}^{4} \cdot e^{4 \alpha r_{s p h}} \cdot \tau_{p} \cdot[t i v s]_{R}^{s p h}}{G_{0, e f f}^{2} \cdot \lambda_{s p h}^{2} \cdot \sigma_{b s, t h e o r y}^{s p h} \cdot\left|\boldsymbol{B}_{i}\left(\theta_{s p h}, \phi_{s p h}\right)\right|^{4} \cdot F_{V V, s p h}^{2} \cdot[t i v s]_{T}} .
$$

Method 2:

$$
\begin{gathered}
\tau_{\text {eff }}=\tau_{\text {eff,old }} \cdot \frac{s_{a, \text { old }}^{\text {sph }}}{s_{a, \text { theory }}^{\text {sph }}} \\
10 \log \left(\frac{\tau_{\text {eff }}}{\tau_{\text {ref }}}\right)=10 \log \left(\frac{\tau_{\text {eff,old }}}{\tau_{r e f}}\right)+10 \log \left(\frac{s_{a, \text { old }}^{\text {sph }}}{s_{a, \text { theory }}^{\text {sph }}}\right) .
\end{gathered}
$$

Method 3:

$$
\begin{gathered}
\tau_{e f f}=\tau_{g} \cdot \frac{G_{0}^{2}}{G_{0, e f f}^{2}} \\
10 \log \left(\frac{\tau_{e f f}}{\tau_{r e f}}\right)=10 \log \left(\frac{\tau_{g}}{\tau_{r e f}}\right)+20 \log \left(\frac{G_{0}}{G_{0, e f f}}\right) .
\end{gathered}
$$

Here, $\tau_{r e f}$ is a reference time interval for $\tau_{e f f}$ (chosen equal to $1 \mathrm{~s}$ ). $\tau_{e f f, \text { old }}$ is an old calibration (or arbitrary, and possibly erroneous) value, used initially for $\tau_{e f f}$ in the calibration procedure.

Equations (63) and (64) for $\sigma_{b s}$ and $s_{v}$, together with Equations (45)-(47) and (68)-(72) for the calibration factors $G_{0, e f f}$ and $\tau_{e f f}$, constitute Formulation $\mathrm{E}$ of the functional relationship for fish abundance estimation.

\section{Results and Discussion}

Four equivalent echo integration Formulations B-E of the power-budget equations for $\sigma_{b s}$ and $s_{v}$ with calibration factors have been derived and described. Formulation $B$ is derived to provide a generic echo integration formulation of the functional relationship for abundance estimation. Formulations C-E have been developed from Formulation B, to investigate the consequences of using "peak voltage echo integration" in single-target TS measurements, as used by some manufacturers [11,15,18-21].

In the following, Formulations A and B are briefly discussed, and Formulations $\mathrm{C}-\mathrm{E}$ are compared to the expressions given for two important and widely used families of echo sounder and sonar systems; (i) the Simrad EK500 [11] and (ii) the Simrad EK60, ES60, ME70, and MS70 [15,18-21] systems.

\subsection{Formulation A (Average Power)}

Formulation A, Equations (1) and (2), derived by [13,16], extends the power-budget equations given in [11] by accounting for arbitrary electrical termination $[13,16]$, represented by an electrical termination factor, $F_{\Pi \Pi}$.

The expressions given in [11] correspond to setting $F_{\Pi}=1$ in Formulation $A$, which is valid for electrical termination conditions for which $Z_{E}=Z_{R}^{*}$ (conjugate matched electrical termination), or for $Z_{E}=Z_{R}$ when $X_{T}=0$ (i.e., in a frequency band close to the series resonance frequency of the transducer vibration mode used) [16]. Otherwise, formulation $A$ is equal to the expressions given for $\sigma_{b s}$ and $s_{v}$ in [11].

As Formulation A, given in terms of average power, is not directly suited for signal processing, it serves here as the basis for deriving the four echo integration formulations, B-E.

\subsection{Formulation B (Echo Integration, Generic)}

Formulation B, Equations (23) and (25) for $\sigma_{b s}$ and $s_{v}$, together with Equations (29) or (31) for the calibration factor $G_{0}$, is derived from Formulation A to provide expressions better suited for practical 
signal processing in echo sounders [16]. This formulation in terms of echo integrals, for calculation of the electrical powers involved, leads to an alternative electrical termination factor in the power-budget equations, $F_{V V}$. It also involves several integration time intervals, $\tau_{p}, \tau_{s t}, \tau_{s p h}$, and $\tau_{g}$, to differentiate between echo integration of the transmitted voltage signal; and echo integration of the single-target (fish), calibration sphere, and volume backscattering voltage echoes, respectively. Formulation B involves only a single calibration factor, $G_{0}$. The generic functional relationship Formulation B constitutes the basis for deriving Formulations $\mathrm{C}-\mathrm{E}$, applicable to "peak voltage echo integration" in single-target $T E$ measurements.

\subsection{Formulation C (Echo Integration, "EK500 Type")}

Formulation C, given by Equations (41) and (42) for $\sigma_{b s}$ and $s_{v}$, together with Equations (45)-(51) for the two calibration factors $G_{0, e f f}$ and $G_{0}$, appears to correspond to, and generalize, the expressions employed in the Simrad EK500 echo sounder [11]. Formulation C is thus for convenience referred to as an "EK500 type" of power-budget equations. This may be seen as follows.

Firstly, the present theory reveals that the expressions for $\sigma_{b s}$ and $s_{v}$ that are actually used in EK500 cannot be exactly those given in its manual [11]. The latter expressions correspond to the average power Formulation A (with $F_{\Pi}=1$ ), whereas the EK500 implementation involves full waveform echo integration for $s_{v}$, "peak voltage echo integration" for single-target TS measurements $\left(\sigma_{b s}\right)$, and two calibration factors "TS transducer gain" and "S $S_{v}$ transducer gain" [11], which neither appear in, nor can be obtained directly from, Formulation A. The echo integration actually used by the manufacturer in the EK500 echo sounder system was never documented in the manual [11] or elsewhere in the available literature. The present theory aims to reveal and explain expressions that appear to be similar to those having been implemented but not documented.

Secondly, by accounting for full waveform echo integration for $s_{v}$, and "peak voltage echo integration" for single-target TS measurements $\left(\sigma_{b s}\right)$ in Formulation C, expressions are here obtained that involve the calibration factors $G_{0, e f f}$ and $G_{0}$. By defining TS transducer gain $\equiv 10 \log _{10}\left(G_{0, e f f}\right)$ and $S_{v}$ transducer gain $\equiv 10 \log _{10}\left(G_{0}\right)$, and using Equation (51), Equations (47) and (50) become identical to the corresponding expressions postulated for the EK500 calibration factors "TS transducer gain" and " $S_{v}$ transducer gain" in [11]. Formulation $C$ is a prerequisite to obtain these expressions.

Consequently, it is shown here that Formulation $C$ leads to expressions for the calibration factors that are identical to those given for the EK500 echo sounder [11]. As these calibration factors cannot be derived directly from the average power budget expressions for $\sigma_{b s}$ and $s_{v}$ given in [11], the analysis shows that the expressions for $\sigma_{b s}$ and $s_{v}$ actually implemented and used in the EK500 system, cannot be those given in [11]. The fact that Formulation $C$ consistently-through the echo integration Formulation B-gives the connection between the average power expressions for $\sigma_{b s}$ and $s_{v}$ given in [11] and these calibration factors, including description of "peak voltage echo integration" in TS measurements, strongly indicates that Formulation C may represent a functional relationship applicable to the EK500 system.

The parameter $G_{0, e f f}$ has been introduced here to account for "peak voltage echo integration" in single-target TS measurements. No such parameter as $G_{0, e f f}$ is used in refs. $[11,15,18-21,23]$.

\subsection{Formulation D (Echo Integration, "EK60 Type")}

Formulation D, given by Equations (52) and (53) for $\sigma_{b s}$ and $s_{v}$, together with Equations (45)-(47) and (58)-(62), respectively, for the two calibration factors $G_{0, e f f}$ and $s_{a, c o r r}$ involved, appears to correspond to, and generalize, the expressions used for the Simrad EK60, ES60, ME70 and MS70 systems [15,18-21]. For convenience, this formulation is thus referred to as an "EK60 type" of power-budget equations. This may be seen as follows.

Firstly, as explained in Section 1, the power-budget equations as these are given in [11] and [15] are not consistent. The ratio of the two different expressions for $s_{v}$ as given by [11] and [15], respectively, is not equal to 1 , but $s_{a, c o r r}^{-2}$. This indicates that the expressions for $\sigma_{b s}$ and $s_{v}$ actually being used in EK60 
and related instruments may be different from those given in [15]. Formulation D is used here to clarify this issue. By accounting for "peak voltage echo integration" for single-target TS measurements in Formulation D, expressions are here obtained that are fully consistent both with [11] and Formulation $C$, and account for $s_{a, c o r r}$ in a consistent way. In Formulations $C$ and D, the expressions for $\sigma_{b s}$ and $s_{v}$ are equivalent, and the ratio of the two expressions for $s_{v}$ equals 1.

Secondly, in Formulation D, expressions are obtained that involve the calibration factors $G_{0, e f f}$ and $s_{a, \text { corr }}$. By defining Gain $\equiv 10 \log _{10}\left(G_{0, \text { eff }}\right)$ and $S_{a}$ correction $\equiv S_{a, \text { corr }} \equiv 10 \log _{10}\left(s_{a, \text { corr }}\right)$, Equations (47) and (62) become equal to expressions used in EK60 [32]. Moreover, the expressions derived here appear to be consistent with the available information given on the "Gain" and " $S_{a}$ correction" calibration factors in the manuals of EK60, etc. [18-21].

The expressions postulated by Ona et al. [15] for $\sigma_{b s}$ and $s_{v}$ correspond to Formulation D. There are, however, some deviations. Firstly (in terms of the terminology used here), in [15], electrical powers $\Pi_{T}$ and $\Pi_{R}$ (in units of $\mathrm{W}$ ) were used instead of echo integrals, $[t i v s]_{T}$, $[\text { tivs }]_{R}^{v}$, and $[\text { tivs }]_{R, \operatorname{st} \text { max }}$ (in units of $\mathrm{V}^{2} \mathrm{~s}=\mathrm{W} \cdot \mathrm{ohm} \cdot \mathrm{s}=\mathrm{J} \cdot \mathrm{ohm}$ ). Echo integration was thus not accounted for in these expressions. Secondly, the same $\Pi_{R}$ was used for $\sigma_{b s}$ and $s_{v}$, which is not consistent with use of "peak voltage echo integration" and full waveform echo integration, respectively, for TS and $s_{v}$ (and thus $s_{A}$ ) measurements. Thirdly, $G(\theta, \phi)$ and $G_{0}$ were used instead of $G_{e f f}(\theta, \phi)$ and $G_{0, e f f}$ in the expressions for $\sigma_{b s}$ and $s_{v}$, respectively, which is inconsistent when "peak voltage echo integration" is used for $\sigma_{b s}$, and $s_{a, \text { corr }}$ is used in $s_{v}$. Finally, in [15], electrical termination was not accounted for, implicitly implying $F_{\Pi}=1[13,16]$, cf. Section 4.1.

In other words, for power-budget equations expressed in terms of echo integrals, and for which "peak voltage echo integration" is used in TS measurements instead of full waveform echo integration, the parameters $G(\theta, \phi)$ and $G_{0}$, referred to in [15] as "transducer gain" and "on-axis transducer gain", respectively, are not equal to the transducer gain and axial transducer gain, $G(\theta, \phi)$ and $G_{0}$, that are involved in the traditional power-budget equation given in [11], as implicitly stated in [15]. Instead, they represent "effective" transducer and axial transducer gains, respectively, $G_{e f f}(\theta, \varphi)$ and $G_{0, e f f}$, with a correction factor $s_{a, \text { corr }}$ involved, to compensate for the use of "peak voltage echo integration" in TS measurements instead of full waveform echo integration, cf. Equations (52) and (53).

Through the derivation of Formulation D, expressions have been obtained that (a) seem to explain and resolve the deviation between the power-budget equations given in [15] and [11]; (b) explain the introduction and use of the $s_{a, c o r r}$ parameter; and (c) "harmonize the TS and $s_{A}$ measurements" [19] by employing the same calibration factor $G_{0, e f f}$ in $\sigma_{b s}$ and $s_{v}$. The power-budget expressions that are consistent with [11], and that may replace those given in [15], appear to be Equations (52) and (53). This includes calibration factors $G_{0, e f f}$ and $s_{a, c o r r}$, given by Equations (45)-(47) and (58)-(62), respectively.

Through the present analysis, the relationship between the Formulation D calibration factors $G_{0, e f f}$ and $s_{a, c o r r}$, and the Formulation $C$ calibration factors, $G_{0, e f f}$ and $G_{0}$, is established, cf. Equation (61). This also includes their relationships to the generic Formulations A and B types of description. It follows that the relationships between the EK500 calibration factors "TS transducer gain" and "S $S_{v}$ transducer gain", and the EK60 etc. calibration factors "Gain" and "S $S_{a}$ correction", also appear to have been established and explained.

As the expressions for $\sigma_{b s}$ and $s_{v}$ derived in [23] correspond exactly to those postulated in [15], the above discussion also applies to [23].

\subsection{On the $G_{e f f}, s_{a, c o r r}$, and $\tau_{e f f}$ Parameters}

The reasons for introducing the parameter $G_{e f f}, s_{a, c o r r}$, and $\tau_{e f f}$ in Formulations C-E, and the need for using two calibration factors for each of these formulations, are explained in the following.

As Formulation B is based on full waveform echo integration both for single-target TS measurements $\left(\sigma_{b s}\right)$ and in survey operation $\left(s_{v}\right)$, only a single calibration factor, $G_{0}$, applies, with no need for $G_{e f f}, s_{a, c o r r}$, and $\tau_{e f f}$ type of parameters. 
In Formulation C, due to the use of "peak voltage echo integration" instead of full waveform echo integration in single-target $T S$ measurements, an error is introduced in $\sigma_{b s}$, which is compensated for by introducing $G_{0, e f f}$ instead of $G_{0}$ in $\sigma_{b s}$, cf. Equations (41), (43), and (44). By still using $G_{0}$ in the expression for $s_{v}$, cf. Equation (42), two calibration factors $G_{0, e f f}$ and $G_{0}$ thus become involved in Formulation C, referred to as "TS transducer gain" and " $S_{v}$ transducer gain", respectively.

In Formulation D, the calibration factor $G_{0}$ used in Formulation $C$ (in the $s_{v}$ expression) is replaced by $G_{0, e f f}$, to "harmonize" the $\sigma_{b s}$ and $s_{v}$ equations. An error is then introduced in $s_{v}$, which is compensated for by using $s_{a, \text { corr }}$ in $s_{v}$, referred to as the " $S_{a}$ correction", cf. Equation (53).

In Formulation E, the term $\tau_{g} s_{a, \text { corr }}^{2}$ used in Formulation D (in the $s_{v}$ equation) is denoted $\tau_{e f f}$. As a result, the $\sigma_{b s}$ and $s_{v}$ expressions of Formulation $\mathrm{E}$ are on the same form as in Formulation B, except that different parameters apply to the two formulations. In Formulation B these are $G(\theta, \phi), G_{0}$, and $\tau_{g}$. In Formulation E these are $G(\theta, \phi), G_{0, e f f}$, and $\tau_{e f f}$, due to the "peak voltage echo integration" used for single-target TS measurements.

In [15] and [23], $\tau_{e f f}$ is referred to as an "effective pulse duration". This interpretation may be discussed. In the present analysis, $s_{a, c o r r}$, used to define $\tau_{e f f}$ in Equation (65), is shown to be essentially a correction factor to compensate for the simplified "peak voltage echo integration" of the received echo in single-target TS measurements, caused by the use of $[t i v s]_{R, \max }^{s t}$ instead of $[t i v s]_{R}^{s t}$ for these targets. Hence, from Equation (65) it appears that $\tau_{e f f}$ represents a scaled gate opening time, to compensate for erroneous echo integration at signal reception for single-target TS measurements. A more representative interpretation of $\tau_{\text {eff }}$ might thus be "effective echo integration time".

\subsection{Comments in Relation to Conventional Operation}

It is emphasized that, from the available literature and practical use in fish abundance estimation, there is no reason to question the expressions actually implemented and used by the manufacturer in the mentioned instruments [11,18-21]. The issues addressed here are all related to the explanation and documentation of the expressions given in the available literature.

It is also emphasized that the derivation and presentation of Formulations B-E by no means indicates that any new method for abundance estimation is proposed, as an alternative to the methods used today. The situation is quite the opposite: The expressions presented here, which are all equivalent, are intended to provide a consistent and unifying theory for improved understanding and control in use of the conventional methods, when employing commonly used echo sounder and sonar systems.

In addition, the more general and complete expressions derived here may constitute an improved basis for evaluation and, if necessary, correction of errors in abundance estimation and species determination. One issue in this respect may be possible system drift, due to changing environment or echo sounder parameters from calibration to oceanic surveying [14]. By knowing the full functional relationship for the abundance measurement, in terms of echo sounder and environmental parameters for the measurement system in question, the possibilities for reliable error evaluation and correction improve.

\section{Conclusions}

On the basis of an average power formulation of power-budget equations for $\sigma_{b s}$ and $s_{v}$ known from prior literature $[13,16]$ (denoted Formulation A), four new echo integration formulations for $\sigma_{b s}$ and $s_{v}$ and associated calibration factors are derived for narrowband signals, denoted Formulations B-E. These are given in terms of the quantities being measured or assumed in calibration and oceanic surveying.

Formulation $B$ gives a generic (instrument independent) functional relationship for fish abundance estimation, and represents a main result of the present work. When waveform integration is used to calculate the echo integrals both in single-target TS measurements and in survey operation, the generic Formulation B serves to be convenient. There is then no need for $G_{e f f}, s_{a, c o r r}$, and $\tau_{\text {eff }}$ type of parameters, and only a single calibration factor $G_{0}$ applies. 
Formulations C-E apply to instruments being based on "peak voltage echo integration" in single-target TS measurements (for calibration, or individual fish). In these formulations, the respective parameters $G_{e f f}, s_{a, c o r r}$, or $\tau_{e f f}$ are introduced to provide consistent and valid functional relationships, and two calibration factors are needed for each formulation. It is a matter of preference which of Formulations C-E is used. Formulations B-E are all equivalent and fully valid functional relationships.

The theory presented here appears to resolve deviations and apparent inconsistencies between various power-budget equations published in prior literature $[11,15,23]$. It is shown that for the $\sigma_{b s}$ and $s_{v}$ expressions given in $[15,23]$ to be consistent with [11], some of the terms involved have to be replaced, to reflect the different "peak voltage echo integration" and full waveform echo integration strategies used for $\sigma_{b s}$ and $s_{v}$, respectively. In terms of the terminology used here, the average electrical powers $\Pi_{T}$ and $\Pi_{R}$ used by $[15,23]$ are to be replaced by echo integrals $[t i v s]_{T}$, $[\text { tivs }]_{R}^{v}$, and $[t i v s]_{R, \max }^{s t}$. Similarly, the transducer gains $G(\theta, \phi)$ and $G_{0}$ used by $[15,23]$ are to be replaced by $G_{e f f}(\theta, \phi)$ and $G_{0, e f f}$. With these modifications applied to the expressions given in [15,23], the expressions given by Formulations B-D for $\sigma_{b s}$ and $s_{v}$ are all consistent with those given in [11,13-16,23]. In terms of this theory, a clear rationale is given for the introduction and use of parameters such as $s_{a, c o r r}$ and $\tau_{e f f}$, which were postulated by [15] and adopted by [23]. $s_{a, c o r r}$ and $\tau_{e f f}$ are here consistently derived and explained.

By comparison with expressions published in available literature it is shown that Formulations $\mathrm{C}$ and D lead to expressions for $\sigma_{b s}, s_{v}$, and calibration factors that appear to correspond to and explain those given for the Simrad EK500 [11], and the Simrad EK60, ES60, ME70, and MS70 systems [15,18-21], respectively. The respective calibration factors involved are here precisely defined. Through Formulations B-C, the mathematical relationship between these calibration factors and the power budget equations given in [11] is established. The power-budget equations for $\sigma_{b s}$ and $s_{v}$ given on echo integration form are expressed directly in terms of the respective calibration factors.

The consistency in the results obtained here indicates that the power-budget equations for $\sigma_{b s}$ and $s_{v}$ employed in the EK500 and the EK60 etc. systems are not exactly those given in [11,15], but rather have forms corresponding to Formulations $\mathrm{C}$ and $\mathrm{D}$, respectively. However, the lack of documentation of the power-budget equations and calibration factors actually used in the EK500, EK60, ES60, ME70, and MS70 systems unfortunately prevents a complete comparison of the expressions derived here for Formulations $\mathrm{C}$ and $\mathrm{D}$ with those used in these systems.

Under the assumption of small-amplitude (linear) sound propagation, the expressions derived here are expected to represent a consistent and relatively complete theoretical basis for improved understanding and control in use of conventional methods and instruments for fish abundance measurement and species identification. Prior literature is extended to provide more complete power-budget equations by accounting for echo integration, electrical termination, and the full range of electrical and acoustical echo sounder parameters. The results are expected to constitute an improved theoretical fundament for measurement, error evaluation, possible error compensation, and uncertainty evaluation of fish abundance methods and equipment in use today.

Acknowledgments: The authors wish to acknowledge Geir Pedersen, Christian Michelsen Research AS, Bergen, Norway; Egil Ona and Gavin J. Macaulay, Institute of Marine Research, Bergen, Norway; and Frank Tichy, Kongsberg Maritime, Horten, Norway, for useful discussions related to topics discussed here.

Author Contributions: The theory (Formulations B-E) was developed by Per Lunde, including description of the relationships between the Formulations A-E, prior literature, and expressions given for scientific echo sounders and sonar systems. Rolf J. Korneliussen contributed with long-time experience from fisheries research surveys and abundance estimation using e.g., the Simrad EK500, EK60, and MS70 systems. Both authors contributed to the assembly and writing of the paper.

Conflicts of Interest: The authors declare no conflict of interest.

\section{References}

1. Clay, C.S.; Medwin, H. Acoustical Oceanography: Principles and Applications; John Wiley \& Sons: New York, NY, USA, 1977; pp. 180-182, 205, 220, 225-235, 395-404. 
2. Nakken, O.; Ulltang, Ø. A Comparison of the Reliability of Acoustic Estimates for Fish Stock Abundance and Estimates Obtained by Other Assessment Methods in Northeast Atlantic; FAO Fish. Rep. No. 300; Food and Agriculture Organization of the United Nations: Rome, Italy, 1983; pp. 249-260.

3. MacLennan, D.N. Acoustical measurements of fish abundance. J. Acoust. Soc. Am. 1990, 87(1), 1-15. [CrossRef]

4. MacLennan, D.N.; Simmonds, E.J. Fisheries Acoustics; Chapman \& Hall: London, UK, 1992; pp. 68-69, 147-151.

5. Medwin, H.; Clay, C.S. Fundamentals of Acoustical Oceanography; Academic Press: Boston, MA, USA, 1998; pp. 153-158, 350-361.

6. Simmonds, J.; MacLennan, D.N. Fisheries Acoustics. Theory and Practice, 2nd ed.; Blackwell Science Ltd.: Oxford, UK, 2005; pp. 32, 47, 59-60, 110, 119-121, 187-191, 197.

7. Dragesund, O.; Olsen, S. On the possibility of estimating year-class strength by measuring echo-abundance of 0-group fish. Fiskeridirektoratets Skrifter, Serie Havundersøkelser 1965, 13(8), 48-75.

8. Dalen, J.; Nakken, O. On the Application of the Echo Integration Method; ICES CM Document No. 1983/B:19; International Council for the Exploration of the Sea: Copenhagen, Denmark, 1983.

9. Foote, K.G. Optimizing copper spheres for precision calibration of hydro acoustic equipment. J. Acoust. Soc. Am. 1982, 71, 742-747. [CrossRef]

10. Foote, K.G.; Knudsen, H.P.; Vestnes, G.; MacLennan, D.N.; Simmonds, E.J. Calibration of Acoustic Instruments for Fish Density Estimation: A Practical Guide; ICES Cooperative Research Report No. 144; International Council for the Exploration of the Sea: Copenhagen, Denmark, 1987.

11. Operator Manual: SIMRAD EK500 Fishery Research Echo Sunder. Scientific Echo Sounder: Base Version. Doc. no. P2170 / Rev. G, Simrad AS (now Kongsberg Maritime AS), Horten, Norway, 1997. pp. 165-169, 204, 211-212, 216-217, 223. Available online: https://www.simrad.com/www/01/NOKBG0397.nsf/AllWeb/ 9F0DB3E3763D8CD3C125718B00332E5A/\$file/043762ag_ek500_operator_manual_english_complete.pdf? OpenElement (accessed on 24 February 2016).

12. Korneliussen, R.J. Analysis and Presentation of Multi-Frequency Echograms. Ph.D. Thesis, University of Bergen, Bergen, Norway, 2002.

13. Pedersen, A. Effects of Nonlinear Sound Propagation in Fisheries Research. Ph.D. Thesis, University of Bergen, Bergen, Norway, 2007. Available online: https://bora.uib.no/handle/1956/2158? mode=full\& submit_simple=Show+full+item+record (accessed on 24 February 2016).

14. Demer, D.A.; Renfree, J.S. Variations in echosounder-transducer performance with water temperature. ICES J. Mar. Sci. 2008, 65, 1021-1035. [CrossRef]

15. Ona, E.; Mazauric, V.; Andersen, L.N. Calibration methods for two scientific multibeam systems. ICES J. Mar. Sci. 2009, 66, 1326-1334. [CrossRef]

16. Lunde, P.; Pedersen, A.O.; Korneliussen, R.J.; Tichy, F.E.; Nes, H. Power Budget and Echo-Integrator Equations for Fish Abundance Estimation. Fisken og Havet no. 10/2013, Institute of Marine Research, Bergen, Norway, 2013. Available online: http://www.imr.no/publikasjoner/andre_publikasjoner/fisken_og_havet/nb-no (accessed on 24 February 2016).

17. MacLennan, D.N.; Fernandes, P.G.; Dalen, J. A consistent approach to definitions and symbols in fisheries acoustics. ICES J. Mar. Sci. 2002, 59, 365-369. [CrossRef]

18. Simrad EK60. Scientific Echo Sounder. Reference Manual. Release 2.4.X. Doc. no. 164692/Rev. D, Kongsberg Maritime AS, Horten, Norway, 2012; pp. 16, 48-49, 191, 199, 221 (in electronic document). Available online: https://www.simrad.com/www/01/nokbg0397.nsf/AllWeb/ 62D6EBE0D8EEB97CC125718E004B41C7?OpenDocument (accessed on 10 July 2016).

19. Simrad ES60. Fish Finding Echo Sounder. Reference Manual. Doc. no. 857-160970 / Rev.H, Kongsberg Maritime AS, Horten, Norway, 2006, p. 80. Available online: http://www.google.no/url?sa=t\&rct=j\& $\mathrm{q}=\&$ esrc=s\&frm $=1 \&$ source=web\&cd=1\&ved=0CCwQFjAA\&url=http $\% 3 \mathrm{~A} \% 2 \mathrm{~F} \% 2 \mathrm{Fwww} . \operatorname{simrad}$. com $\%$ 2Fwww\%2F01\%2FNOKBG0397.nsf\%2FAllWeb\%2F476DD263FD01A618C12570D100630F2B\%2F\%24file\% 2F160970ah_es60_reference_manual_english.pdf\%3FOpenElement\&ei=W4-EU9m1D4SB4gSn8IHoAw\& usg=AFQjCNH8YxK7LuYNCspKNZ5g9bSlu8PgJA\&sig2=V4kxvlh0jDwMNNDKxQ7pGQ\&bvm=bv. 67720277,d.bGE (accessed on 24 February 2016). 
20. Simrad ME70. Scientific Multibeam Echo Sounder. Reference Manual. Release 1.2.5. Doc. no. 328588 / Rev. B, Kongsberg Maritime AS, Horten, Norway, 2012, pp. 140, 143, 152, 154, 158, 210, 246, 448 (in electronic document). Available online: http:/ / www.simrad.com/www/01/nokbg0397.nsf/AllWeb/ DCB276EA00D5BD46C125719A003AAD45?OpenDocument (accessed on 24 February 2016).

21. Simrad MS70. Scientific Multibeam Sonar. Operator MANUAL. Doc. no. 301088 / Rev. C, Kongsberg Maritime AS, Horten, Norway, 2007, pp. 164, 171, 179 . Available online: http://www.google. no/url?sa $=$ t\&rct=j\&q=\&esrc $=$ s\&frm $=1 \&$ source $=$ web\&cd $=1 \&$ ved $=0 C C 8 Q F j A A \& u r l=h t t p \% 3 A \% 2 F \%$ 2Fwww.imr.no\%2Ffilarkiv\%2F2006\%2F08\%2F301088ac_ms70_operator_manual_english.pdf\%2Fnnno\&ei=65OEU_OeLoPm4QTB9IHYBQ\&usg=AFQjCNEMV_2RNtMRZSqEXuo1QAuZkj3h5A\&sig2= WRfF6vgyljMjYk7yUVthkA\&bvm=bv.67720277,d.bGE (accessed on 24 February 2016).

22. Lunde, P.; Korneliussen, R.J. A Unifying Theory Explaining Different Power-Budget Formulations Used in Modern Scientific Echo Sounders for Fish Abundance Estimation. Fisken og Havet no. 7/2014, Institute of Marine Research, Bergen, Norway, 2014. Available online: http://www.imr.no/publikasjoner/andre_publikasjoner/ fisken_og_havet/nb-no (accessed on 24 February 2016).

23. Calibration of Acoustic Instruments. ICES Cooperative Research Report No. 326, International Council for Exploration of the Sea: Copenhagen, Denmark, May 2015, pp. 12-16, 62. Available online: http://www.google.no/url?sa=t\&rct=j\&q=\&esrc=s\&source=web\&cd=1\&ved= 0ahUKEwjdyLWZrJDLAhWh93IKHVU9CpoQFgggMAA\&url=http\%3A\%2F\%2Fices.dk\%2Fsites\% 2Fpub\%2FPublication\%2520Reports\%2FCooperative\%2520Research\%2520Report\%2520(CRR) \%2Fcrr326\%2FCRR326.pdf\&usg=AFQjCNHpagCdqvZT8zLLQCVcKB8Jej-XFQ\&bvm=bv.114733917, d.bGQ (accessed on 24 February 2016).

24. Korneliussen, R.J.; Diner, N.; Ona, E.; Berger, L.; Fernandes, P.G. Proposals for the collection of multifrequency acoustic data. ICES J. Mar. Sci. 2008, 65, 982-994. [CrossRef]

25. Tichy, F.E.; Solli, H.; Klaveness, H. Nonlinear effects in a $200-\mathrm{kHz}$ sound beam and consequences for target strength measurement. ICES J. Mar. Sci. 2003, 60, 571-574. [CrossRef]

26. Lunde, P.; Pedersen, A.O. Sonar and Power-Budget Equations for Backscattering of Finite Amplitude Sound Waves, with Implications in Fishery Acoustics for Abundance Estimation of Marine Resources. In Proceeding of 35th Scand. Symp. Phys. Acoust., Geilo, Norway, 29 January-1 Feburary 2012. Available online: http:/ / www.ntnu.edu/sspa/program (accessed on 24 February 2016).

27. Foote, K.G. Rather-high-frequency sound scattering by swimbladdered fish. J. Acoust. Soc. Am. 1985, 78, 688-700. [CrossRef]

28. Foote, K.G. Linearity of fisheries acoustics, with addition theorems. J. Acoust. Soc. Am. 1983, 73(6), $1932-1940$. [CrossRef]

29. Balanis, C.A. Antenna Theory: Analysis and Design, 3rd ed.; John Wiley \& Sons: Hoboken, NJ, USA, 2005; pp. 50, 66, 89-95.

30. Kinsler, L.E.; Frey, A.R.; Coppens, A.B.; Sanders, J.V. Fundamentals of Acoustics, 4th ed.; John Wiley \& Sons: New York, NY, USA, 2000; pp. 15, 181-184,189.

31. Brigham, E.O. The Fast Fourier Transform; Prentice-Hall: Englewood Cliffs, NJ, USA, 1974; p. 64.

32. Ona, E. (Institute of Marine Research, Bergen, Norway). Personal communication, 2014.

(C) 2016 by the authors; licensee MDPI, Basel, Switzerland. This article is an open access article distributed under the terms and conditions of the Creative Commons Attribution (CC-BY) license (http://creativecommons.org/licenses/by/4.0/). 